\title{
Effect of combined light-emitting diodes on the accumulation of glucosinolates in Brassica microgreens
}

\author{
Oday Alrifai ${ }^{1,2}$, Lili Mats', Ronghua Liu', Xiuming Hao ${ }^{3}$, Massimo F. Marcone ${ }^{2}$ and Rong Tsao ${ }^{1 *}$ (D)
}

\begin{abstract}
As of recent, microgreen vegetable production in controlled environments are being investigated for their bioactive properties. Phytochemicals like glucosinolates (GLS) are highly sensitive to varying spectral qualities of light, especially in leafy greens of Brassica where the responses are highly species-dependent. The accumulation of bioactive GLS were studied under 8 different treatments of combined amber $(590 \mathrm{~nm})$, blue $(455 \mathrm{~nm})$, and red $(655$ $\mathrm{nm}$ ) light-emitting diodes (rbaLED). A semi-targeted metabolomics approach was carried out to profile common intact-GLS in microgreen extracts of Brassica by means of LC-HRMS/MS. Thirteen GLS were identified, among them were 8 aliphatic, 4 indolic and 1 aromatic GLS. Mass spectrometry data showed sinigrin had the highest average concentration and was highest in B. juncea, progoitrin was highest in B. rapa and glucobrassicin in $R$. sativus. The individual and total GLS in the microgreens of the present study were largely different under rbaLED; $B$. rapa microgreens contained the highest profile of total GLS, followed by $R$. sativus and $B$. juncea. Sinigrin was increased and gluconasturtiin was decreased under rbaLED lighting in most microgreens, glucoalyssin uniquely increased in $R$. sativus and decreased in B. rapa and glucobrassicin uniquely decreased in both B. rapa and B. juncea. The present study showed that rbaLED contributed to the altered profiles of GLS resulting in their significant modulation. Optimizing the light spectrum for improved GLS biosynthesis could lead to production of microgreens with targeted health-promoting properties.
\end{abstract}

Keywords: Amber light, Glucosinolates, Light-emitting diodes, Microgreens, Controlled environment agriculture, Phytochemical, Controlled environment systems

\footnotetext{
* Correspondence: rong.cao@agr.gc.ca

${ }^{1}$ Guelph Research \& Development Center, Agriculture and Agri-Food Canada,

93 Stone Road West, Guelph, Ontario N1G 5C9, Canada

Full list of author information is available at the end of the article
}

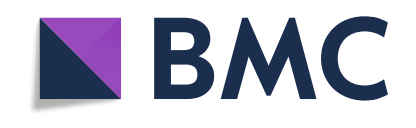

(c) The Author(s). 2021 Open Access This article is licensed under a Creative Commons Attribution 4.0 International License, which permits use, sharing, adaptation, distribution and reproduction in any medium or format, as long as you give appropriate credit to the original author(s) and the source, provide a link to the Creative Commons licence, and indicate if changes were made. The images or other third party material in this article are included in the article's Creative Commons licence, unless indicated otherwise in a credit line to the material. If material is not included in the article's Creative Commons licence and your intended use is not permitted by statutory regulation or exceeds the permitted use, you will need to obtain permission directly from the copyright holder. To view a copy of this licence, visit http://creativecommons.org/licenses/by/4.0/. 


\section{Graphical Abstract}

\section{Light-emitting diodes ( $\mathrm{nm}$ )}
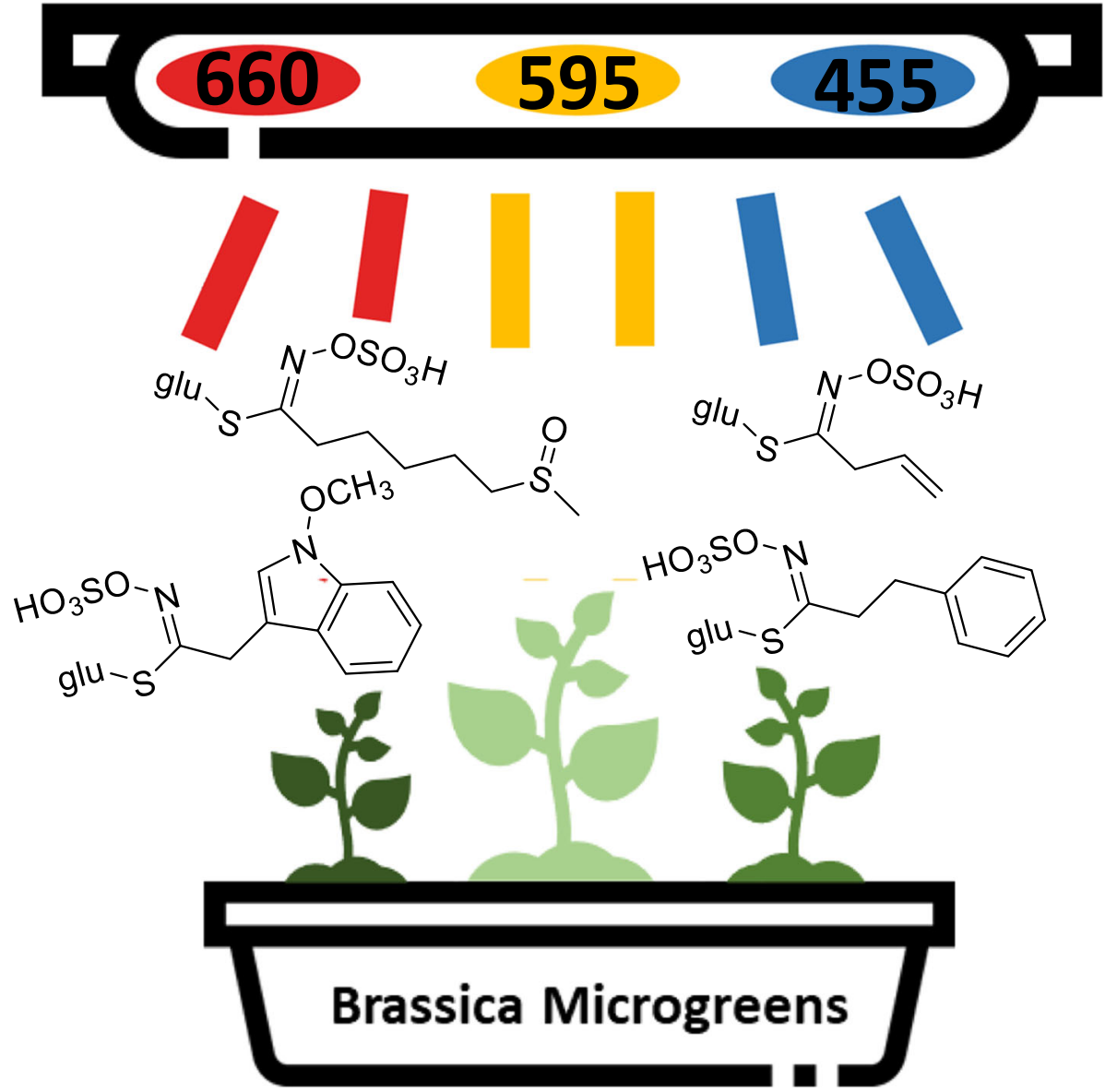

\section{Introduction}

Glucosinolates (GLS) are hydrophilic and sulfurcontaining plant secondary metabolites with over 130 variants, and are particularly found in leafy Brassica vegetables and seeds. Plant GLS and their metabolites are recognized for their fungicidal, nematocidal and bactericidal properties (Dekić et al. 2017; Sotelo et al. 2015; Yu et al. 2007). Medical research is also exploring their modulatory roles in chronic cardiovascular disease and certain cancers (Fahey et al. 2001). Due to their abundant and diverse chemical substituents, GLS are classified according to their functional framework i.e. allyl, indole and aromatic types from the respective precursors methionine, phenylalanine and tryptophan. Although the primary function of plant GLS are not known, tissue disruption initiates a myrosinase-catalyzed breakdown which yields glucose, sulfate and the aglycone. The aglycone is subsequently rearranged to give produce the bioactive defense-related mustard oil aroma against biotic factors (Au-Grosser and Au-van-Dam 2017). The pungent flavors of GLS-containing Brassica vegetables are imparted by allyl isothiocyanate, the main breakdown product of sinigrin and a candidate for anticarcinogenic and chemoprotective studies (Zhang 2010). It is the ratio between GLS and their breakdown products that dictates the distinct aroma and flavor of Brassica (Maina et al. 2020). GLS also breakdown to other bioactives including nitriles, epithionitriles thiocyanates and indoles, which are also shown to possess beneficial biological activities (Hahn et al. 2016). Due to the complexity of GLS in various Brassica vegetables and their instability and lack of commercial reference materials, identification and analysis of these compounds has been primarily done using LC-MS on intact-GLS (i.e. glycosylated GLS). 
Medical studies have highlighted the usefulness of combined GLS and their breakdown products for supplementary health benefits, especially for antiinflammatory and antioxidant purposes. There is a general agreement to which higher GLS exist in young seedlings and sprouts on a per gram basis in many Brassicas compared to their mature counterparts, thus leading to promoting the consumption of microgreens (Tan et al. 2020). Biological activity of GLS are a function of their breakdown products, with minor activities imparted by intact GLS; however, the bioavailability of more than one compound may enhance the synergistic functions which are largely dictated by genetic composition and enzyme activity (Maina et al. 2020). GLS breakdown products (i.e. isothiocyanate, indole-3-carbinols and nitriles) have been studied for their efficacy as antimicrobial, chemo-preventative and anti-inflammatory agents (Barba et al. 2016; Maina et al. 2020).

Recently, there has been interest for improving the composition of intact GLS or their breakdown products, especially in various genotypes, by understanding the metabolomic, transcriptomic and proteomic profiles related to their biosynthesis. Some studies have already investigated genes of Brassica controlling GLS biosynthesis in cabbage, turnip, broccoli and pac choi, especially those grown in controlled environments under novel light-emitting diodes (LED) wavelengths (Chun et al. 2018; Kim et al. 2017; Wang et al. 2021; Yan et al. 2020; Yang et al. 2020; Zuluaga et al. 2019).

Light (spectral) quality play a large role in GLS metabolism; however, the effects are largely understudied in microgreens of Brassica (Carvalho and Folta 2014; Chun et al. 2018; Kopsell et al. 2015; Lee et al. 2016; Mølmann et al. 2020; Moon et al. 2015; C. H. Park et al. 2019; Park et al. 2020; Rechner et al. 2017). GLS biosynthesis of various Brassica vegetables have been reported under LED; however, there is limited availability of studies detailing novel combinations of LED light in the visible spectrum, such as supplementary amber light (AL) to basal red light (RL) and blue light (BL). Additionally, no other study has approached a targeted analysis using LC-MS for the changes in GLS under varying fractions of AL. The aim of this work is to carry out a semitargeted metabolomics approach to profile common intact-GLS in Brassica microgreen extracts by means of LC-HRMS/MS. Under various ratios of combined red light $(660 \mathrm{~nm})$, blue light $(455 \mathrm{~nm})$ and $\mathrm{AL}(590 \mathrm{~nm})$ (rbaLED), the amount of decreasing RL was adjusted accordingly to increasing AL to maintain a similar photosynthetic active range and to ensure non-confounding effects between spectral quality and quantity (Snowden et al. 2016). This new information will provide further insight into the effect of AL on bioactives in Brassica microgreens, in addition to the phenolics and carotenoids, to understand narrow-band LED effects (Alrifai et al. 2020, 2021).

\section{Materials and methods \\ Plant materials, growth chamber lighting and chemical reagents}

Eight varieties of Brassica microgreens: mizunas (organic (MO), Brassica rapa var. Japonica; red kingdom (MR); Pac choi (PC), Brassica rapa var. Chinensis; radishes (red Rambo (RR) and red Rambo organic (RO), Raphanus sativus), and mustards (Scarlet Frills (MSF), Barbarossa (MB) and Garnet Giant (MG), Brassica juncea) were grown at the Harrow Research \& Development Centre, Agriculture and Agri-Food Canada (Harrow, Ontario, Canada). Similar to our previous reports, rbaLED in various combinations were used to grow the microgreens over two separate trials (Table S1, Additional file 1) (Alrifai et al. 2020, 2021). Durations for seed germination and growth in the chambers for each microgreen are listed in Table 1 . Microgreens were harvested at their base, packed in air-tight plastic bags and stored in $-80^{\circ} \mathrm{C}$ until analysed shortly afterwards. Details related to growth conditions and sample extraction have been reported in our previous paper (Alrifai et al. 2020).

Pure standards of sinigrin, glucobrassicin and gluconasturtiin were purchased from Sigma-Aldrich (St. Louis, MO, USA). HPLC grade solvents of methanol $(\mathrm{MeOH})$, formic acid and acetonitrile $(\mathrm{ACN})$ were purchased from Thermo Fisher Scientific (Waltham, MA, USA). All chemical reagents used were of analytical grade. Samples of each microgreen species were analyzed separately using LC-MS. Peak area averages of the extracted ion current of individual GLS were determined from triplicates. Representative chromatograms depicting the relative abundance (\%) of GLS species in the samples are shown in Fig. 1. Following the initial semi-targeted analysis, 13 GLS of interest were selected and quantified (Fig. 2). Based on structural similarity, sinigrin was used to quantify allyl GLS; glucobrassicin, indole GLS and gluconasturtiin, aromatic GLS. Concentrations of GLS in each group were expressed as the equivalencies of the corresponding standard, and were calculated using the calibration curves $\left(R^{2}>0.99\right)$ generated from serial dilutions between 0.01 to $30 \mu \mathrm{g} / \mathrm{mL}$ (Fig. 3).

\section{Statistical analysis}

One-way ANOVA followed by Dunnett's post hoc test $(p<0.05)$ was used for all GLS from each microgreen under all the lighting treatments using IBM SPSS software for Windows version 25.0 (IBM corporation, Armonk, NY, USA). Relative standard deviation (RSD) from the untargeted peak area analysis was $<5 \%$. 
Table 1 Concentration of glucosinolates in each genus B. rapa, B. juncea and R. sativus under all combined treatments of amber, red and blue LED ${ }^{a}$

\begin{tabular}{|c|c|c|c|c|c|c|c|c|c|c|c|}
\hline & \multicolumn{4}{|l|}{ B. juncea ${ }^{b}$} & \multicolumn{4}{|l|}{ B. rapa $^{c}$} & \multicolumn{3}{|l|}{ R. sativus ${ }^{d}$} \\
\hline & Average $^{\mathrm{e}}$ & MB & MG & MSF & Average $^{\mathrm{e}}$ & MO & MR & PC & Average $^{\mathrm{e}}$ & RO & RR \\
\hline Seed germination $(d)^{f}$ & 2 & & & & 2 & & & & 2 & & \\
\hline Growth chamber $(d)^{f}$ & 13 & & & & 14 & & & & 13 & & \\
\hline Seed to harvest $(d)^{f}$ & 15 & & & & 16 & & & & 15 & & \\
\hline Glucosinolate & \multicolumn{11}{|c|}{ average concentration ( $\mu \mathrm{g} / \mathrm{g}$ dry weight) } \\
\hline Sinigrin & 793.6 & 670.4 & 829.9 & 880.6 & 124.3 & 65.2 & 238.3 & 69.5 & 34.2 & 3.7 & 64.7 \\
\hline Glucoiberin $^{9}$ & 22.0 & 40.6 & 11.1 & 14.2 & - & - & - & - & 19.4 & 22.7 & 16.0 \\
\hline Glucoraphanin ${ }^{9}$ & 1.1 & - & 1.1 & - & 133.2 & 97.9 & 130.4 & 171.4 & 72.1 & 72.3 & 72.0 \\
\hline Progoitrin $^{f}$ & 9.1 & 9.2 & 8.6 & 9.6 & 1029.0 & 929.0 & 1187.3 & 970.7 & 2.1 & 2.9 & 0.9 \\
\hline Gluconapin ${ }^{9}$ & 314.7 & 263.1 & 496.2 & 184.8 & 301.6 & 401.2 & 234.6 & 269.2 & 8.2 & 8.1 & 8.4 \\
\hline Glucoerucin ${ }^{9}$ & 3.4 & 3.7 & 1.2 & 3.7 & 809.9 & 502.7 & 999.4 & 927.6 & 86.3 & 84.1 & 88.4 \\
\hline Glucobrassicin & 324.7 & 131.9 & 296.2 & 546.0 & 770.5 & 695.2 & 841.6 & 774.8 & 1425.6 & 1378.1 & 1473.0 \\
\hline Glucoalyssin ${ }^{9}$ & 1.3 & 1.1 & 1.4 & - & 162.7 & 227.1 & 164.2 & 96.8 & 7.1 & 7.1 & 7.1 \\
\hline 4-Methoxyglucobrassicin ${ }^{h}$ & 198.8 & 233.0 & 193.7 & 169.8 & 293.4 & 249.3 & 233.0 & 397.8 & 320.7 & 324.5 & 316.8 \\
\hline 4-Hydroxyglucobrassicin ${ }^{h}$ & 243.2 & 131.6 & 266.6 & 331.3 & 181.2 & 131.7 & 267.6 & 144.2 & 495.6 & 479.6 & 511.5 \\
\hline Gluconapoleiferin ${ }^{9}$ & - & - & - & - & 48.9 & 62.8 & 55.8 & 28.3 & - & - & - \\
\hline Neoglucobrassicin ${ }^{h}$ & 186.1 & 193.7 & 222.9 & 141.7 & 890.5 & 1151.0 & 654.7 & 865.8 & 7.5 & 7.1 & 8.0 \\
\hline Gluconasturtiin & 131.2 & 123.1 & 98.9 & 171.6 & 320.1 & 264.0 & 398.8 & 297.6 & 1.6 & 1.5 & 1.8 \\
\hline Total (sum) Glucosinolates & 2229.2 & 1801.4 & 2427.8 & 2453.3 & 5065.3 & 4777.1 & 5405.7 & 5013.7 & 2480.4 & 2391.7 & 2568.6 \\
\hline
\end{tabular}

${ }^{a}$ Only one extraction replica was used for quantification, as the semi-targeted analysis revealed high fidelity (RSD $\left.<5 \%\right)$ of extracted samples in all microgreens tested; ${ }^{b}$ mustard (Barbarossa (MB), Scarlett Frills (MSF) and Garnet Giant (MG)); ' mizuna (Red Kingdom (MR) and Organic (MO)), Pac Choi (Red Pac (PC)); ${ }^{d}$ radish (Red Rambo (RR) and Red Rambo Organic (RO)); ${ }^{\mathrm{e}}$ microgreens of the same genus only; ${ }^{\mathrm{f}}$ days for seed germination, growth in chamber and total duration from seed to harvest; contents expressed as ${ }^{\mathrm{g}}$ sinigrin and ${ }^{\mathrm{h}}$ glucobrassicin equivalents

\section{Glucosinolates analysis by liquid chromatography-high resolution tandem mass spectrometry (LC-HRMS/MS)}

An untargeted profiling approach combining full MS and MS/MS methods was used to obtain reliable peak areas and fragmentation data in one run. Pooled quality control (QC) samples, one for each microgreen species, were created by combining $10 \mu \mathrm{L}$ of each sample. Extracted samples in triplicate were randomized and injected in FullMS mode; QC samples were periodically inserted into sequence and injected in DDMS2 mode. LC-HRMS/MS analysis was performed using a Thermo ${ }^{\circ}$ Scientific Q-Exactive ${ }^{\mathrm{rm}}$ Orbitrap mass spectrometer equipped with a Vanquish ${ }^{\mathrm{TM}}$ Flex Binary UPLC System (Waltham, MA, USA). Data were acquired using Thermo Scientific $^{\mathrm{TM}}$ Xcalibur $^{\mathrm{TM}} 4.2$ software and Thermo Scientific $^{\mathrm{Tm}}$ Standard Integration Software. The chromatographic separation was performed on a Kinetex XBC18 100A HPLC column $(100 \times 4.6 \mathrm{~mm}, 2.6 \mu \mathrm{m}$, Phenomenex Inc., Torrance, CA, USA). The binary mobile phase consisted of solvent A $\left(99.9 \% \mathrm{H}_{2} \mathrm{O} / 0.1 \%\right.$ formic acid) and solvent $\mathrm{B}(94.9 \% \mathrm{MeOH} / 5 \% \mathrm{ACN} / 0.1 \%$ formic acid). The following solvent gradient was used: 0-5 min, 0 to $12 \% \mathrm{~B} ; 5-15 \mathrm{~min}, 12$ to $23 \% \mathrm{~B}$; $15-30 \mathrm{~min}, 23$ to $50 \% \mathrm{~B} ; 30-40 \mathrm{~min}, 50$ to $80 \% \mathrm{~B} ; 40-42 \mathrm{~min}, 80$ to $100 \% \mathrm{~B}$; $42-45 \mathrm{~min}, 100 \% \mathrm{~B}$; $45-46 \mathrm{~min}, 100$ to $0 \% \mathrm{~B}$;
46-52 min, 0\% B. The column compartment temperature was held at $40{ }^{\circ} \mathrm{C}$, the flow rate was set at $0.700 \mathrm{~mL} / \mathrm{min}$ and the injection volume was $1 \mu \mathrm{L}$ for the extracted samples ( 1 or $5 \mu \mathrm{L}$ for the QC samples). MS data was collected in negative ionization mode (spray voltage $=2.8 \mathrm{kV}$ ) with scan range set to $90-1300 \mathrm{~m} / \mathrm{z}$. Extracted samples were analyzed in FullMS mode, and QC samples were analyzed with DDMS2 (Top $N=15$ ) method, with NCE set at 30. Details on LOD and LOQ for sinigrin, glucobrassicin and gluconasturtiin in each microgreen are listed in Table S2 (Additional file 2). The same FullMS settings were used for quantification part of the analysis, where dilutions of the standard mixture were run together with one set of extracts for each microgreen species. Only one extraction replica was used for quantification, as the semi-targeted analysis revealed high fidelity ( $\mathrm{RSD}<5 \%)$ of extracted samples in all microgreens tested. Phytochemical screening and quantitation have also been conducted using HPLC and LC-MS for crude plant extracts using single sample injections (Choi et al. 2018; El Sayed et al. 2020; Keskes et al. 2017). Data was visualized and analysed using Thermo FreeStyle ${ }^{\mathrm{TM}} 1.6$ software. 


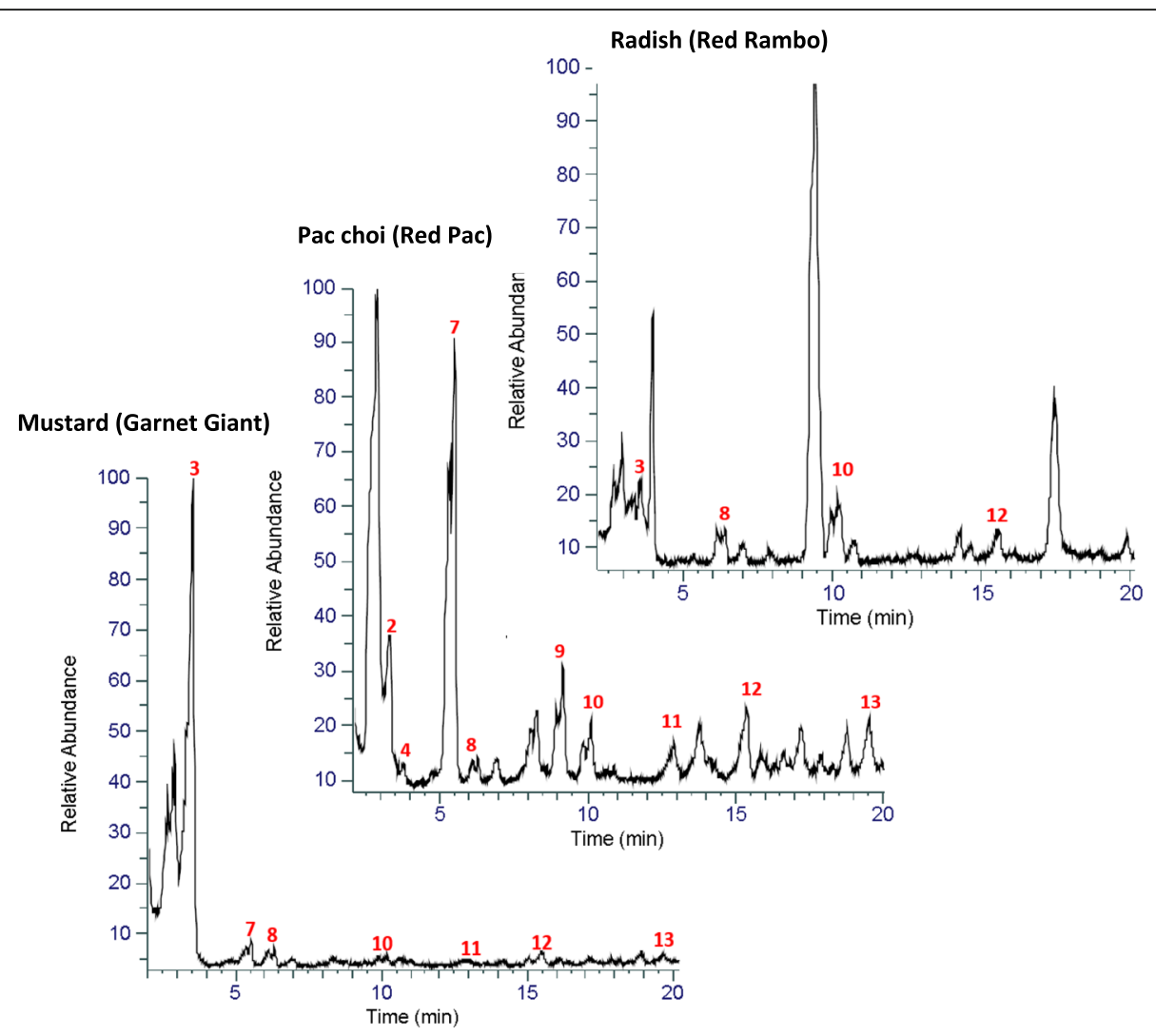

Fig. 1 Relative peak abundance (\%) of the extracted ion chromatograms of glucosinolates extracts of representative Brassica microgreens [B. rapa (Pac choi), B. juncea (mustard) and R. sativus (radish)]. Compounds are 2 progoitrin; 3 sinigrin; 4 glucoraphanin; 7 gluconapin; 8 4hydroxyglucobrassicin; 9 glucoerucin; 10 glucobrassicin; 11, gluconasturtiin; 12, 4-methoxyglucobrassicin; 13, neoglucobrassicin

\section{Results and discussion}

\section{Identification of Glucosinolates}

Identification of GLS were based on accurate mass data $\mathrm{m} / \mathrm{z}$, retention time and fragmentation pattern. Identification and quantification of GLS was done in the same run (relative quantification), which allowed us to look at trends and identify multitude of compounds at the same time. A total of 13 GLS were selected from the eight microgreens grown under rbaLED, based on peak intensity (ion abundance) and reported bioactive properties, and were identified by comparing MS/MS fragmentation with literature data, and that of the standards (for the 3 GLS standards). The 13 GLS peaks were identified as: $\mathrm{C}_{11} \mathrm{H}_{21} \mathrm{NO}_{10} \mathrm{~S}_{3}$ glucoiberin, 1; $\mathrm{C}_{11} \mathrm{H}_{19} \mathrm{NO}_{10} \mathrm{~S}_{2}$ progoitrin, 2; $\mathrm{C}_{10} \mathrm{H}_{16} \mathrm{KNO}_{9} \mathrm{~S}_{2}$ sinigrin, 3; $\mathrm{C}_{12} \mathrm{H}_{23} \mathrm{NO}_{10} \mathrm{~S}_{3}$ glucoraphanin, 4; $\mathrm{C}_{12} \mathrm{H}_{21} \mathrm{NO}_{10} \mathrm{~S}_{2}$ gluconapoleiferin, 5; $\mathrm{C}_{13} \mathrm{H}_{25} \mathrm{NO}_{10} \mathrm{~S}_{3}$ glucoalyssin, 6; $\quad \mathrm{C}_{11} \mathrm{H}_{19} \mathrm{NO}_{9} \mathrm{~S}_{2}$ gluconapin, 7; $\mathrm{C}_{16} \mathrm{H}_{20} \mathrm{~N}_{2} \mathrm{O}_{10} \mathrm{~S}_{2}$ 4-hydroxyglucobrassicin, 8; $\mathrm{C}_{12} \mathrm{H}_{23} \mathrm{NO}_{9} \mathrm{~S}_{3}$ glucoerucin, 9; $\mathrm{C}_{16} \mathrm{H}_{20} \mathrm{~N}_{2} \mathrm{O}_{9} \mathrm{~S}_{2}$ glucobrassicin, 10; $\mathrm{C}_{15} \mathrm{H}_{21} \mathrm{NO}_{9} \mathrm{~S}_{2}$ gluconasturtiin, 11; $\mathrm{C}_{17} \mathrm{H}_{22} \mathrm{~N}_{2} \mathrm{O}_{10} \mathrm{~S}_{2}$ 4methoxyglucobrassicin, 12; and $\mathrm{C}_{17} \mathrm{H}_{22} \mathrm{~N}_{2} \mathrm{O}_{10} \mathrm{~S}_{2}$ neoglucobrassicin, 13 (Table 2).

Negative ionization MS is widely used for GLS analysis. The sulfate moiety allows for facile ionization, and the resulting predictable fragmentation also allows for selectively targeting GLS in complex samples. In the present study, in addition to the deprotonated molecular GLS ion, we applied MS/MS to confirm the presence of several characteristic fragments for identification. Normalized collision energy of 30 used in MS/MS experiments resulted in abundant S-containing fragments $\mathrm{HSO}_{4}{ }^{-} \quad(\mathrm{m} / \mathrm{z}=96.9610), \quad \mathrm{SO}_{4}{ }^{\cdot-} \quad(\mathrm{m} / \mathrm{z}=95.9522)$, $\left.\mathrm{OHC}_{2} \mathrm{H}_{2} \mathrm{~S}^{-}(\mathrm{m} / \mathrm{z})=74.9910\right)$ and $\mathrm{SO}_{3}{ }^{-}(\mathrm{m} / \mathrm{z}=79.9574)$. Most GLS MS/MS spectra also contained thioglucosebased fragments, such as $\mathrm{C}_{6} \mathrm{H}_{11} \mathrm{O}_{6} \mathrm{SO}_{3}{ }^{-}(\mathrm{m} / \mathrm{z}=$ 259.0129), $\quad \mathrm{SHC}_{6} \mathrm{H}_{10} \mathrm{O}_{5} \mathrm{SO}_{3}{ }^{-} \quad(\mathrm{m} / \mathrm{z}=274.9900)$ and $\mathrm{C}_{6} \mathrm{H}_{11} \mathrm{O}_{5} \mathrm{~S}^{-}(\mathrm{m} / \mathrm{z}=195.0333)$. Another minor fragment $\mathrm{m} / \mathrm{z}=119.0350$ is ubiquitous to all GLS tested; it can be attributed to glucose fragmentation, resulting into the $\mathrm{C}_{4} \mathrm{H}_{7} \mathrm{O}_{4}{ }^{-}$anion (Table 2) (Bialecki et al. 2010). Some of the unique fragments in each GLS can be attributed to a common combined loss of sulfur trioxide and neutral loss of a glucose moiety $\left(\mathrm{C}_{6} \mathrm{H}_{10} \mathrm{O}_{8} \mathrm{~S}^{-} \mathrm{m} / \mathrm{z}=242.0102\right)$ (Clarke 2010; Maldini et al. 2012). These include $\mathrm{m} / \mathrm{z}=$ 115.9280 (sinigrin), $\mathrm{m} / \mathrm{z}=178.0368$ (glucoerucin), $\mathrm{m} / \mathrm{z}=$ 205.0445 (glucobrassicin) and $\mathrm{m} / \mathrm{z}=208.0473$ (glucoalyssin). The neutral loss of $\mathrm{CH}_{3} \mathrm{SOH}(\mathrm{m} / \mathrm{z}=63.9988)$ is common to GLS with methylsulfinyl group, where 


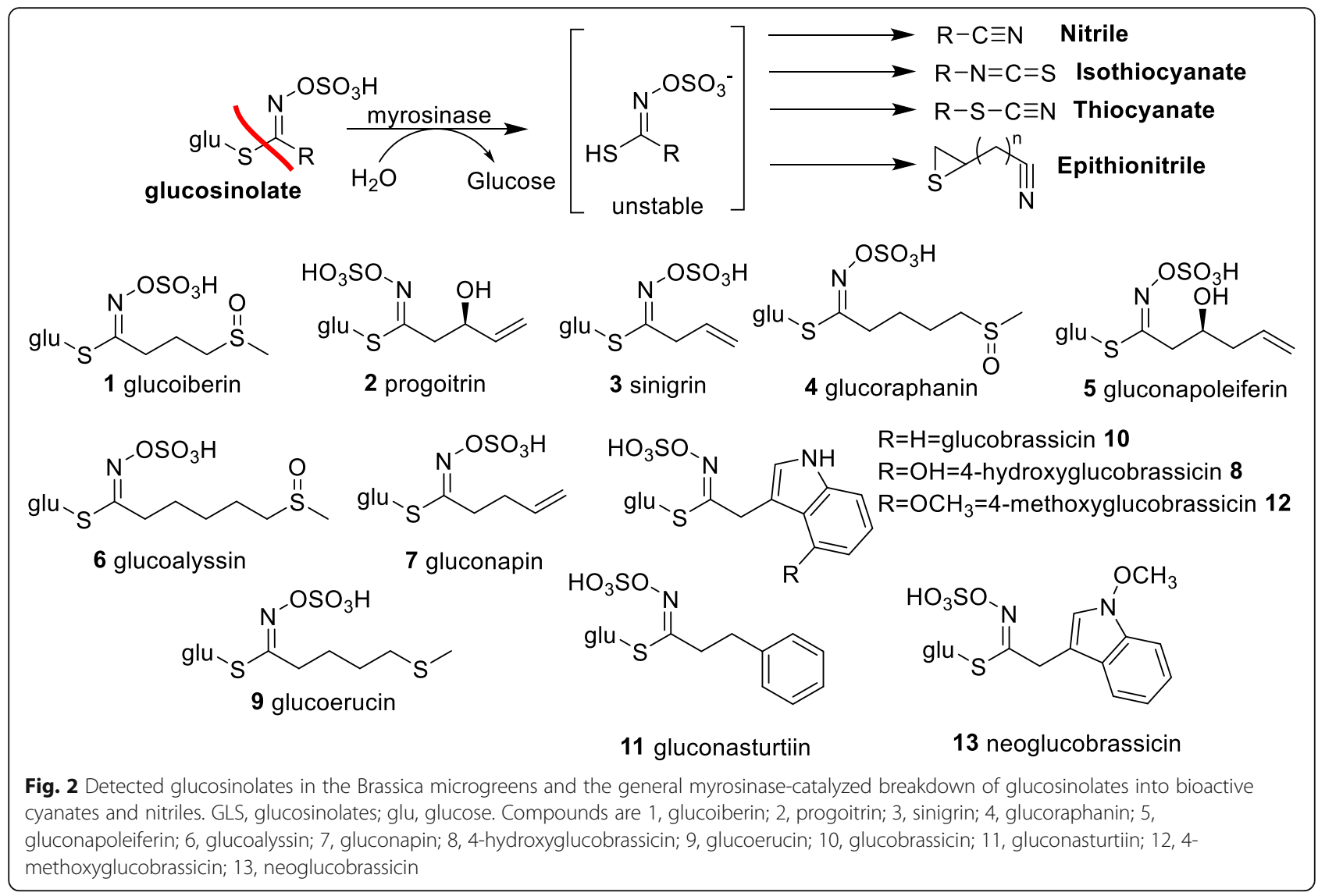

unique fragments are observed in glucoiberin $(\mathrm{m} / \mathrm{z}=$ 358.0284) and glucoalyssin $(\mathrm{m} / \mathrm{z}=386.0587)$ (Shi et al. 2017). Neoglucobrassicin and the isomer 4methoxyglucobrassicin share the identical parent ion; however, their fragmentation spectra were distinct. MS/ MS spectrum of 4-methoxyglucobrassin showed higher intensity of fragments with $\mathrm{m} / \mathrm{z}>100$ including the precursor ion, whereas neoglucobrassicin fragmented into low-mass fragments only. Neither fragmentation spectra contained the commonly reported methoxy radical loss product ( $\mathrm{m} / \mathrm{z} 446)$; however, neoglucobrassicin showed a unique major product ion $\mathrm{m} / \mathrm{z} 154.0543$ (Maldini et al. 2012; Shi et al. 2017). Final decision on 4methoxyglucobrassin vs neoglucobrassicin assignment was done based on retention time (Table 2) (Bhandari et al. 2020; Hooshmand and Fomsgaard 2021).

\section{Effect of rbaLED on Glucosinolates}

The individual and total GLS in the microgreens of the present study were largely different under rbaLED (Tables 1 and 3). B. rapa microgreens contained the highest profile of total GLS, followed by $R$. sativus and $B$. juncea. Sinigrin was increased under rbaLED lighting in most microgreens, glucoalyssin uniquely increased in $R$. sativus and decreased in B. rapa and glucobrassicin uniquely decreased in both $B$. rapa and B. juncea.(Table $1)$. Gluconasturtiin was decreased in most microgreens under rbaLED and was the least concentrated GLS in $R$. sativus. All GLS but glucoiberin were found in B. rapa microgreens at relatively high concentrations. Glucosinolate profiles of $B$. juncea and $R$. sativus were less diverse and the concentrations were overall lower compared to those in B. rapa (Table 1). Despite these apparent differences in total GLS of the 8 microgreens, the effects by rbaLED on GLS at each treatment level were more complicated, and further analysis showed that certain individual GLS were significantly modulated under rbaLED treatments and their responses were species-specific (Table 3). The effects of rbaLED on the individual GLS (aliphatic GLS: compounds 1-7, 9; indole and aromatic GLS: 8, 10-13) in the 8 microgreens are discussed below.

\section{Aliphatic GLS}

Among the aliphatic short chain-GLS, sinigrin 3 was mostly found in B. juncea; however, its concentration was affected variably by the rbaLED depending on the microgreen variety. Sinigrin in B. juncea was significantly decreased under rbaLED treatments in MB (2.524.1\%) and MSF (0.7-31\%), but increased in MO 
$Y=-26713.8+6.14851 e+006^{*} X \quad R^{\wedge} 2=0.9951 W: 1 / X^{\wedge} 2$

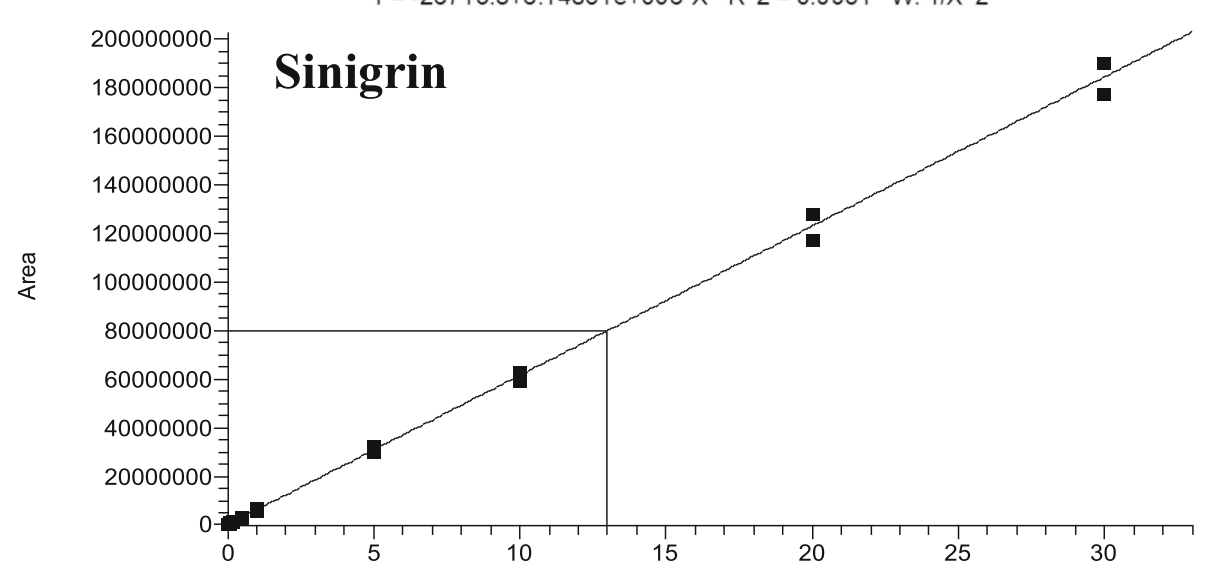

$Y=-75841.6+3.05728 e+006^{*} X \quad R^{\wedge} 2=0.9961 W: 1 / X^{\wedge} 2$

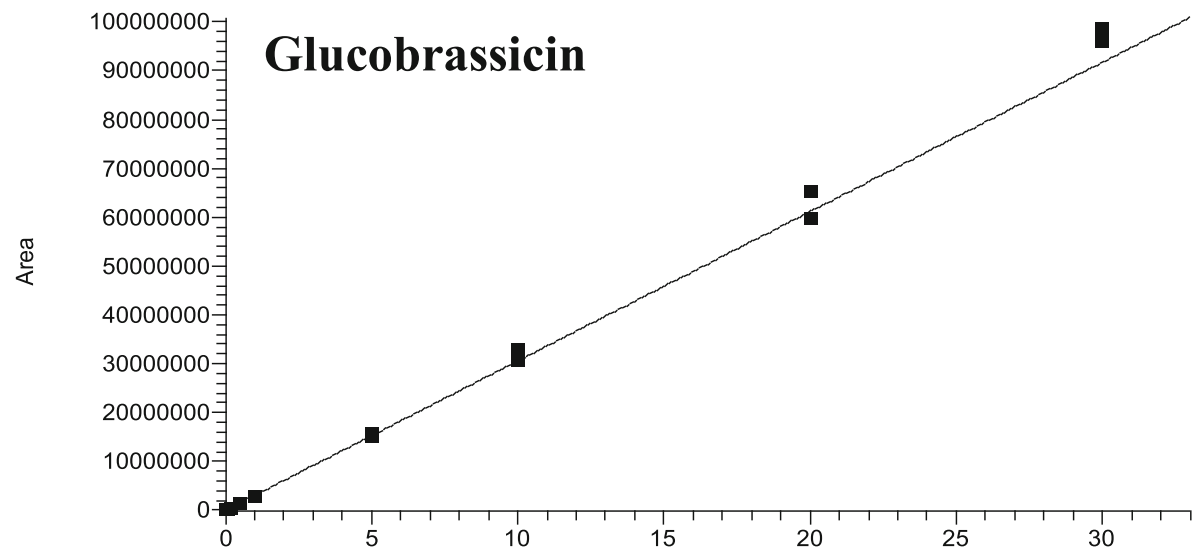

$Y=-117566+8.09364 e+006^{*} X \quad R^{\wedge} 2=0.9980 W: 1 / X^{\wedge} 2$

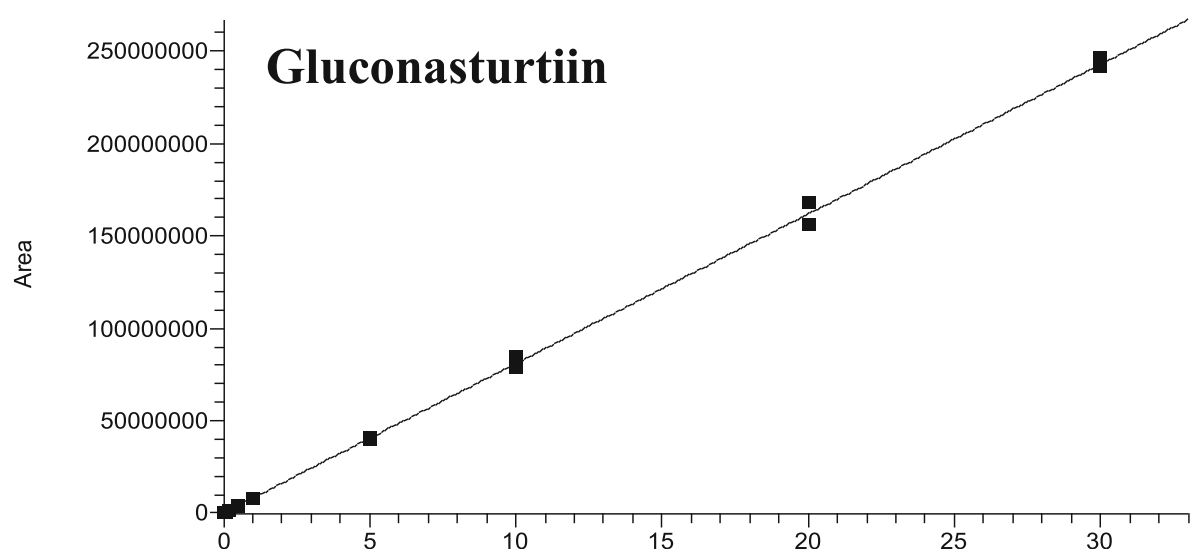

Fig. 3 Calibration curves $\left(R^{2}>0.99\right)$ obtained by running a three-standard mixture of sinigrin, glucobrassicin and gluconasturtiin with the concentration range of 0.01 to $30 \mu \mathrm{g} / \mathrm{mL}$

(736.5-1591.7\%), MG (6.6-13.9\%), PC (39.7-928.6\%), RO (44.4-2000\%) and RR (255.6-7616.7\%) MR (30.293.1\%) under treatments T2-T8 compared to the control T1 (Table 3). In a study on B. juncea sprouts, sinigrin was increased under red and white LED and decreased under blue LED, an observation that contrasted our results in which we see a significant decrease in sinigrin in microgreens of MB, MG and MSF (Chang Ha Park et al. 2020). Generally in B. juncea (MB, MG and MSF), higher sinigrin was found in compared to $B$. rapa 
Table 2 Glucosinolates identified by LC-HRMS/MS. ${ }^{a}$

\begin{tabular}{lllll}
\hline$\#$ & Glucosinolate & $\begin{array}{l}\text { Retention } \\
\text { time, } \mathbf{m i n}\end{array}$ & $\begin{array}{l}\text { Precursor } \\
\text { ion, } \mathbf{~} / \mathbf{z}\end{array}$ & Fragment ions, m/z \\
\hline $\mathbf{1}$ & Glucoiberin & 2.91 & 422.0257 & $96.9604,95.9526,74.9911,79.9575,195.0336,358.0276,259.0125$ \\
$\mathbf{2}$ & Progoitrin & 3.32 & 388.0379 & $96.9604,95.9526,74.9911,79.9575,195.0336,135.9713,259.0129,274.9904$ \\
$\mathbf{3}$ & Sinigrin & 3.5 & 358.0272 & $96.9601,74.9910,79.9574,195.0331,161.9866,116.0176,259.0127,274.9901$ \\
$\mathbf{4}$ & Glucoraphanin & 3.77 & 436.0413 & $96.9604,95.9526,74.9911,79.9575,178.0183,372.0430,195.0331,259.0133$ \\
$\mathbf{5}$ & Gluconapoleiferin & 5.02 & 402.0534 & $96.9604,95.9526,74.9911,79.9575,195.0336,160.0443,259.0115,274.9905$ \\
$\mathbf{6}$ & Glucoalyssin & 5.18 & 450.0569 & $96.9604,95.9526,74.9911,79.9575,192.0339,195.0341,386.0587,259.0143,274.9910$ \\
$\mathbf{7}$ & Gluconapin & 5.51 & 372.0430 & $96.9604,95.9526,74.9911,79.9575,195.0336,178.9847,259.0128,274.9903$ \\
$\mathbf{8}$ & 4-Hydroxyglucobrassicin & 6.37 & 463.0486 & $96.9604,95.9526,74.9911,79.9575,195.0334,221.0391,267.008122285 .0184$ \\
$\mathbf{9}$ & Glucoerucin & 9.32 & 420.0462 & $96.9605,74.9911,178.0369,195.0334,259.01315,274.9902,226.9877$ \\
$\mathbf{1 0}$ & Glucobrassicin & 10.33 & 447.0537 & $96.9605,95.9527,74.9911,79.9576,259.0128,274.9902,205.0445,195.0336$ \\
$\mathbf{1 1}$ & Gluconasturtiin & 13.1 & 422.0587 & $96.9604,95.9526,74.9911,79.9575,259.0127,180.0490,195.0335,274.9902,229.0001$ \\
$\mathbf{1 2}$ & 4-Methoxyglucobrassicin & 15.68 & 477.0643 & $96.9603,95.9525,74.9910,79.9574,259.0119,274.9899,235.0546,195.0338,119.0350$ \\
$\mathbf{1 3}$ & Neoglucobrassicin & 19.82 & 477.0643 & $96.9605,95.9527,74.9912,79.9574,154.0543,259.0127,170.0490,205.0443,282.9848$
\end{tabular}

${ }^{a}$ Only one extraction replica was used for quantification, as the semi-targeted analysis revealed high fidelity (RSD $\left.<5 \%\right)$ of extracted samples in all microgreens tested

microgreens with trace amounts, a result similarly found in other Brassica vegetables (Rangkadilok et al. 2002). Sinigrin concentration in microgreens of $B$. oleracea (broccoli) was not significantly affected by monochromatic blue LED and combined red and blue LED and combined red, blue and green LED, suggesting a clear species-specific response and dependence on LED regimes for sinigrin biosynthesis in Brassica microgreens, and the need for fine-tuning the light spectrum to improve its accumulation (Kopsell and Sams 2013). Increased sinigrin content in microgreens by LED may have added health benefits as studies have demonstrated therapeutic effects of this unique phytochemical, thus further investigation is needed (Cartea and Velasco 2008; Mazumder et al. 2016). LED lighting appears to affect the biosynthesis of GLS by altering the expression of certain genes. A recent study showed that red LED increased the aliphatic GLS biosynthesis of broccoli seedlings by upregulating the expression of SOT18 compared to the white light, red light and combined red and blue light, and the indole GLS by upregulating the CYP family of genes through enhancing the tryptophan content. (Wang et al. 2021). Fine-tuning the light spectrum to modulate certain genes that participate in the GLS biosynthetic pathway would ultimately lead to the development of microgreens with high contents of aliphatic GLS, such as sinigrin, which is a known healthpromoting compound.

The precursor of sinigrin, glucoiberin 1, was overall significantly decreased in MB (18.5-44.6\%) and MSF (14-66.2\%), but increased in RO (2.6-101.6\%) and variably affected in MG and RR (Table 3). Under rbaLED treatments in all microgreens, the total concentration of glucoiberin was lowest $(837 \mu \mathrm{g} / \mathrm{g})$ among the detected GLS (837-49,093 $\mu \mathrm{g} / \mathrm{g}$ ) (Table 1). Sinigrin and glucoiberin were generally decreased in the microgreens under rbaLED, although other studies on different Brassica microgreens grown under different LED conditions (broccoli microgreens under combined red and blue LED and monochromatic blue LED, and kale under red LED) showed no significant effects (Kopsell and Sams 2013; Lefsrud et al. 2008). Glucoiberin was not significantly different in $B$. juncea vegetables under white, red or blue LED, while higher sinigrin was correlated with exposure duration (Park et al. 2020). In B. napus, glucoiberin was also not significantly changed under white, red, blue or combined red and blue LED; however, sinigrin was significantly increased under only red LED compared to the other lights (Park et al. 2019). Optimizing the light spectrum and photoperiod to produce desirable outcomes for sinigrin and glucoiberin in Brassica species should be further investigated.

Glucoraphanin 4 was found in all three species of microgreens of the present study. Compared to the $B$. rapa and $R$. sativus microgreens, glucoraphanin was only found in very low concentration in MG $(1.1 \mu \mathrm{g} / \mathrm{g}$ d.w. (dry weight)) among the B. juncea microgreens (Table 1). Glucoraphanin was significantly increased overall under rbaLED in MO (11-261.7\%), PC (23-218.2\%), RR (18.1-118.9\%) and RO (9.8-36.8\%). MG under the treatments T4 and T5 contained higher quantities of glucoraphanin compared to trace amounts in control plants (T1) (Table 3). Other studies have found no or trace amounts of glucoraphanin in Brassica microgreens (Liu et al. 2012; Rangkadilok et al. 2002). Previous reports have shown that the accumulation of methylsulfinylalkyl 
Table 3 Concentration of the glucosinolates identified in the microgreens under combined red, blue and amber LED. ${ }^{a}$ Values are expressed as $\mu \mathrm{g}$ sinigrin $^{\mathrm{b}}$, glucobrassicin ${ }^{c}$ or gluconasturtiin ${ }^{\mathrm{d}}$ equivalents per $\mathrm{g}$ of microgreens (dry weight)

\begin{tabular}{|c|c|c|c|c|c|c|c|c|c|}
\hline \multirow[b]{2}{*}{ Glucosinolate } & \multirow[b]{2}{*}{ LED $^{\mathbf{e}}$} & \multicolumn{3}{|c|}{ B. juncea } & \multicolumn{3}{|l|}{ B. rapa } & \multicolumn{2}{|c|}{ R. sativus } \\
\hline & & $\overline{M B}$ & MG & MSF & MO & MR & PC & $\mathrm{RO}$ & RR \\
\hline \multirow[t]{8}{*}{ Sinigrin } & T1 & 745.9 & 837.4 & 931.5 & 9.6 & 451.3 & 23.4 & 0.9 & 1.8 \\
\hline & $\mathrm{T} 2$ & 731.5 & 836.2 & $701.1^{*}$ & $162.4^{*}$ & $315.0^{*}$ & $32.7^{*}$ & $4.2^{*}$ & $118.9^{*}$ \\
\hline & T3 & $727.0^{*}$ & $893^{*}$ & $925.3^{*}$ & $80.3^{*}$ & $224.6^{*}$ & $173.9^{*}$ & 0.5 & $97.3^{*}$ \\
\hline & T4 & $566.4^{*}$ & $902.9^{*}$ & $884.2^{*}$ & 13.0 & $31.3^{*}$ & $33.7^{*}$ & 1.4 & $81.9^{*}$ \\
\hline & T5 & $634.1^{*}$ & $697.8^{*}$ & 1001.3 & 9.9 & $169.7^{*}$ & $11.9^{*}$ & $1.3^{*}$ & 1.7 \\
\hline & T6 & 658.6 & $953.5^{*}$ & $1009^{*}$ & $143.1^{*}$ & $222.9^{*}$ & 24.4 & 1.2 & $70.6^{*}$ \\
\hline & T7 & $655.0^{*}$ & $815.5^{*}$ & 950.1 & $92.0^{*}$ & $297.2^{*}$ & $240.7^{*}$ & $18.9^{*}$ & $138.9^{*}$ \\
\hline & T8 & $644.4^{*}$ & $702.5^{*}$ & $642.6^{*}$ & 11.0 & $194.4^{*}$ & $15.5^{*}$ & 1.4 & $6.4^{*}$ \\
\hline \multirow[t]{8}{*}{ Glucoiberin $^{c}$} & $\mathrm{~T} 1$ & 53.6 & 12.5 & 20.7 & ND & ND & ND & 19.3 & 16.3 \\
\hline & $\mathrm{T} 2$ & $36.7^{*}$ & $11.0^{*}$ & $12.1^{*}$ & ND & ND & ND & 20.0 & $17.8^{*}$ \\
\hline & T3 & $43.7^{*}$ & 12.0 & $17.8^{*}$ & ND & ND & ND & $20.2^{*}$ & $13.8^{*}$ \\
\hline & T4 & $29.7^{*}$ & $9.3^{*}$ & $8.9^{*}$ & ND & ND & ND & $20.4^{*}$ & 14.2 \\
\hline & T5 & $38.8^{*}$ & $12.5^{*}$ & $16.2^{*}$ & ND & ND & ND & $19.8^{*}$ & $15.6^{*}$ \\
\hline & T6 & $40.2^{*}$ & $13.1^{*}$ & $14.8^{*}$ & ND & ND & ND & $22.2^{*}$ & $14.1^{*}$ \\
\hline & T7 & $41.9^{*}$ & $12.5^{*}$ & $16.1^{*}$ & ND & ND & ND & $38.9^{*}$ & $18.8^{*}$ \\
\hline & T8 & $40.5^{*}$ & $5.7^{*}$ & $7.0^{*}$ & ND & ND & ND & $20.9^{*}$ & $17.6^{*}$ \\
\hline \multirow[t]{8}{*}{ Glucoraphanin $^{c}$} & $\mathrm{~T} 1$ & trace & trace & trace & 62.1 & 61.2 & 105.1 & 58.9 & 60.8 \\
\hline & $\mathrm{T} 2$ & trace & trace & ND & $82.7^{*}$ & 105.4 & 243.3 & 61.8 & 60.4 \\
\hline & T3 & ND & ND & ND & $92.4^{*}$ & 187.0 & $129.3^{*}$ & $68.9^{*}$ & 58.3 \\
\hline & T4 & trace & 0.5 & ND & 68.2 & 182.7 & 135.3 & $64.7^{*}$ & 52.0 \\
\hline & T5 & ND & 1.6 & trace & $68.9^{*}$ & 139.7 & 94.0 & $80.6^{*}$ & $71.8^{*}$ \\
\hline & T6 & ND & trace & trace & $97.1^{*}$ & 198.1 & 203.0 & 77.5 & 64.2 \\
\hline & T7 & trace & trace & trace & 87.3 & 50.8 & $334.4^{*}$ & 90.0 & $75.6^{*}$ \\
\hline & T8 & ND & ND & ND & $224.6^{*}$ & 118.6 & $126.7^{*}$ & $75.7^{*}$ & $133.1^{*}$ \\
\hline \multirow[t]{8}{*}{ Progoitrin $^{c}$} & $\mathrm{~T} 1$ & 9.7 & 5.7 & 14.1 & 950.0 & 1186.2 & 1076.1 & 2.2 & ND \\
\hline & $\mathrm{T} 2$ & $9.8^{*}$ & $8.7^{*}$ & $8.8^{*}$ & $863.0^{*}$ & $1085.7^{*}$ & $1106.0^{*}$ & ND & 0.6 \\
\hline & T3 & $12.8^{*}$ & $6.0^{*}$ & $6.2^{*}$ & $863.3^{*}$ & $1127.6^{*}$ & $757.8^{*}$ & ND & ND \\
\hline & $\mathrm{T} 4$ & 8.6 & $7.7^{*}$ & $8.2^{*}$ & $866.8^{*}$ & $1150.0^{*}$ & $815.3^{*}$ & ND & ND \\
\hline & T5 & $5.8^{*}$ & $6.5^{*}$ & $13.6^{*}$ & $1040.1^{*}$ & $1580.0^{*}$ & $581.6^{*}$ & 2.2 & ND \\
\hline & T6 & $5.0^{*}$ & $7.6^{*}$ & $12.3^{*}$ & $971.1^{*}$ & $1330.5^{*}$ & $1239.3^{*}$ & ND & ND \\
\hline & T7 & $12.3^{*}$ & $15.7^{*}$ & $7.0^{*}$ & $961.3^{*}$ & $1181.1^{*}$ & $1415.6^{*}$ & 4.4 & 1.1 \\
\hline & T8 & $9.4^{*}$ & $10.8^{*}$ & $6.4^{*}$ & $916.3^{*}$ & $857.1^{*}$ & $773.7^{*}$ & ND & ND \\
\hline \multirow[t]{8}{*}{ Gluconapin ${ }^{c}$} & $\mathrm{~T} 1$ & 311.0 & 461.0 & 233.6 & 411.1 & 247.7 & 288.1 & 33.3 & 0.6 \\
\hline & $\mathrm{T} 2$ & $285.1^{*}$ & 482.1 & $133.1^{*}$ & $377.6^{*}$ & $218.4^{*}$ & $325.3^{*}$ & $1.4^{*}$ & $18.9^{*}$ \\
\hline & T3 & $295.4^{*}$ & $512.5^{*}$ & $178.8^{*}$ & $371.0^{*}$ & $243.9^{*}$ & $235.4^{*}$ & $1.6^{*}$ & $4.2^{*}$ \\
\hline & $\mathrm{T} 4$ & $258.6^{*}$ & 654.6 & 214.2 & 416.0 & 215.6 & 211.1 & 2.0 & 3.7 \\
\hline & T5 & $224.9^{*}$ & 435.0 & 189.6 & 477.8 & 289.1 & 164.7 & 21.1 & 1.1 \\
\hline & T6 & $222.2^{*}$ & $512.6^{*}$ & $241.3^{*}$ & $418.0^{*}$ & $242.8^{*}$ & $372.2^{*}$ & $3.0^{*}$ & $1.3^{*}$ \\
\hline & T7 & $261.6^{*}$ & $514.4^{*}$ & $172.6^{*}$ & $396.0^{*}$ & $268.0^{*}$ & $303.2^{*}$ & $1.1^{*}$ & $35.9^{*}$ \\
\hline & T8 & $245.6^{*}$ & $397.3^{*}$ & $114.8^{*}$ & $341.7^{*}$ & $151.0^{*}$ & $253.4^{*}$ & $1.4^{*}$ & $1.1^{*}$ \\
\hline \multirow[t]{2}{*}{ Glucoerucin ${ }^{c}$} & $\mathrm{~T} 1$ & 3.5 & ND & 4.0 & 819.5 & 1151.8 & 1056.6 & 83.6 & 90.3 \\
\hline & T2 & $5.9^{*}$ & 1.2 & $2.2^{*}$ & $437.4^{*}$ & $729.7^{*}$ & $1283.9^{*}$ & $69.7^{*}$ & 88.8 \\
\hline
\end{tabular}


Table 3 Concentration of the glucosinolates identified in the microgreens under combined red, blue and amber LED. ${ }^{a}$ Values are expressed as $\mu \mathrm{g}$ sinigrin $^{\mathrm{b}}$, glucobrassicin ${ }^{\mathrm{c}}$ or gluconasturtiin ${ }^{\mathrm{d}}$ equivalents per $\mathrm{g}$ of microgreens (dry weight) (Continued)

\begin{tabular}{|c|c|c|c|c|c|c|c|c|c|}
\hline \multirow[b]{2}{*}{ Glucosinolate } & \multirow[b]{2}{*}{ LED $^{e}$} & \multicolumn{3}{|c|}{ B. juncea } & \multicolumn{3}{|l|}{ B. rapa } & \multicolumn{2}{|c|}{ R. sativus } \\
\hline & & $\overline{M B}$ & MG & MSF & MO & MR & PC & RO & RR \\
\hline & T3 & $2.6^{*}$ & ND & ND & $593.1^{*}$ & $924.0^{*}$ & 881.4 & $84.3^{*}$ & 95.5 \\
\hline & $\mathrm{T} 4$ & $2.3^{*}$ & ND & trace & $490.4^{*}$ & $1201.6^{*}$ & 966.8 & $106.9^{*}$ & 82.4 \\
\hline & T5 & ND & ND & 3.9 & $485.3^{*}$ & 1161.1 & $670.9^{*}$ & $100.0^{*}$ & $129.1^{*}$ \\
\hline & T6 & ND & ND & $4.5^{*}$ & $608.9^{*}$ & $1368.0^{*}$ & $1147.5^{*}$ & $79.9^{*}$ & $85.4^{*}$ \\
\hline & T7 & $4.1^{*}$ & ND & ND & 356.7 & $870.5^{*}$ & $791.1^{*}$ & $103.9^{*}$ & $100.3^{*}$ \\
\hline & T8 & ND & ND & ND & $230.1^{*}$ & $588.7^{*}$ & $622.7^{*}$ & $44.6^{*}$ & $35.3^{*}$ \\
\hline \multirow[t]{8}{*}{ Glucobrassicin } & $\mathrm{T} 1$ & 160.7 & 322.5 & 561.5 & 786.0 & 983.0 & 912.6 & 1111.4 & 1666.6 \\
\hline & $\mathrm{T} 2$ & $153.4^{*}$ & $312.3^{*}$ & $529.5^{*}$ & $712.3^{*}$ & $872.9^{*}$ & $896.7^{*}$ & 1215.5 & $1547.3^{*}$ \\
\hline & T3 & $141.3^{*}$ & $332.5^{*}$ & $593.8^{*}$ & $683.2^{*}$ & $915.4^{*}$ & $641.0^{*}$ & 1375.6 & $1558.6^{*}$ \\
\hline & $\mathrm{T} 4$ & $110.2^{*}$ & 359.8 & 540.4 & 674.6 & $815.9^{*}$ & $697.4^{*}$ & $2031.2^{*}$ & $1566.5^{*}$ \\
\hline & T5 & $126.0^{*}$ & $260.6^{*}$ & $516.9^{*}$ & 771.4 & $826.4^{*}$ & 711.3 & 1307.8 & $1688.2^{*}$ \\
\hline & T6 & $124.9^{*}$ & 305.6 & $667.7^{*}$ & 669.6 & $1028.0^{*}$ & $970.4^{*}$ & $1394.6^{*}$ & $1490.6^{*}$ \\
\hline & T7 & $127.7^{*}$ & 314.7 & 609.2 & $772.7^{*}$ & $858.8^{*}$ & $677.9^{*}$ & $1251.7^{*}$ & $1208.5^{*}$ \\
\hline & T8 & $111.2^{*}$ & $161.2^{*}$ & $348.7^{*}$ & $491.9^{*}$ & $432.2^{*}$ & 691.1 & $1337.2^{*}$ & 1057.5 \\
\hline \multirow[t]{8}{*}{ Glucoalyssin ${ }^{c}$} & $\mathrm{~T} 1$ & ND & $\mathrm{ND}$ & trace & 219.2 & 145.1 & 91.1 & 5.8 & 6.0 \\
\hline & $\mathrm{T} 2$ & ND & 1.2 & ND & $189.9^{*}$ & $131.3^{*}$ & 116.1 & 5.1 & $6.5^{*}$ \\
\hline & T3 & ND & trace & ND & $210.5^{*}$ & 229.9 & 86.6 & 6.4 & $7.2^{*}$ \\
\hline & $\mathrm{T} 4$ & 1.1 & trace & trace & $210.2^{*}$ & 239.6 & 91.3 & $7.5^{*}$ & 5.8 \\
\hline & T5 & ND & trace & ND & $214.1^{*}$ & 150.6 & $61.9^{*}$ & $5.2^{*}$ & $6.7^{*}$ \\
\hline & T6 & ND & trace & ND & $226.1^{*}$ & 238.0 & 122.6 & 6.0 & 6.4 \\
\hline & T7 & trace & 1.6 & ND & 230.0 & 94.6 & $131.4^{*}$ & $8.9^{*}$ & $8.6^{*}$ \\
\hline & T8 & ND & trace & ND & $316.9^{*}$ & $84.4^{*}$ & $73.2^{*}$ & $12.2^{*}$ & $9.6^{*}$ \\
\hline \multirow[t]{8}{*}{ 4-Methoxyglucobrassicin ${ }^{d}$} & $\mathrm{~T} 1$ & 251.2 & 180.0 & 156.6 & 252.7 & 282.5 & 381.5 & 395.2 & 341.9 \\
\hline & $\mathrm{T} 2$ & $250.4^{*}$ & 185.4 & $176.0^{*}$ & $261.5^{*}$ & $199.5^{*}$ & $424.7^{*}$ & $266.3^{*}$ & 339.1 \\
\hline & T3 & $213.2^{*}$ & $185.5^{*}$ & $140.4^{*}$ & $254.8^{*}$ & $241.2^{*}$ & $428.2^{*}$ & $260.9^{*}$ & $295.6^{*}$ \\
\hline & $\mathrm{T} 4$ & $258.1^{*}$ & $258.1^{*}$ & $182.8^{*}$ & $261.0^{*}$ & 289.2 & $439.0^{*}$ & $439.2^{*}$ & $338.7^{*}$ \\
\hline & T5 & $188.1^{*}$ & 178.3 & $171.4^{*}$ & 266.2 & $219.3^{*}$ & $361.6^{*}$ & 231.5 & 298.8 \\
\hline & T6 & $237.9^{*}$ & $184.3^{*}$ & $170.3^{*}$ & $221.2^{*}$ & $208.4^{*}$ & $452.6^{*}$ & $284.2^{*}$ & $303.8^{*}$ \\
\hline & T7 & $228.9^{*}$ & $192.9^{*}$ & 164.2 & $255.3^{*}$ & $233.5^{*}$ & $350.0^{*}$ & $276.1^{*}$ & $266.6^{*}$ \\
\hline & T8 & $236.2^{*}$ & $184.7^{*}$ & $197.0^{*}$ & $221.9^{*}$ & $190.7^{*}$ & $344.4^{*}$ & $442.6^{*}$ & $350.0^{*}$ \\
\hline \multirow[t]{8}{*}{ 4-Hydroxyglucobrassicin ${ }^{d}$} & $\mathrm{~T} 1$ & 168.4 & 281.0 & 357.4 & 143.4 & 330.5 & 166.6 & 412.7 & 558.6 \\
\hline & $\mathrm{T} 2$ & 140.3 & 272.9 & $279.9^{*}$ & $141.9^{*}$ & $248.7^{*}$ & $190.5^{*}$ & $498.3^{*}$ & 558.0 \\
\hline & T3 & $120.7^{*}$ & $310.0^{*}$ & $333.6^{*}$ & $138.1^{*}$ & $271.8^{*}$ & $152.6^{*}$ & $457.1^{*}$ & $479.5^{*}$ \\
\hline & T4 & 139.3 & $329.8^{*}$ & $335.7^{*}$ & 150.0 & $294.4^{*}$ & 131.5 & $523.6^{*}$ & 508.3 \\
\hline & T5 & $83.4^{*}$ & $217.0^{*}$ & $331.8^{*}$ & $129.1^{*}$ & $258.5^{*}$ & $94.0^{*}$ & $555.6^{*}$ & 499.1 \\
\hline & T6 & $155.7^{*}$ & $289.4^{*}$ & $416.8^{*}$ & $138.3^{*}$ & $335.2^{*}$ & $202.3^{*}$ & $453.0^{*}$ & $506.7^{*}$ \\
\hline & T7 & $112.4^{*}$ & $287.0^{*}$ & $329.0^{*}$ & $132.7^{*}$ & $279.6^{*}$ & $130.1^{*}$ & 491.1 & $537.5^{*}$ \\
\hline & T8 & $132.9^{*}$ & $145.3^{*}$ & $266.5^{*}$ & 79.9.0* & $121.7^{*}$ & $86.2^{*}$ & $445.3^{*}$ & 444.6 \\
\hline \multirow[t]{4}{*}{ Gluconapoleiferin ${ }^{c}$} & $\mathrm{~T} 1$ & ND & ND & ND & 58.7 & 62.2 & 38.9 & ND & ND \\
\hline & $\mathrm{T} 2$ & ND & ND & ND & $73.3^{*}$ & $48.0^{*}$ & 34.1 & ND & ND \\
\hline & T3 & ND & ND & ND & $55.5^{*}$ & $55.3^{*}$ & $25.1^{*}$ & ND & ND \\
\hline & $\mathrm{T} 4$ & ND & ND & ND & $58.3^{*}$ & 61.1 & $22.7^{*}$ & ND & ND \\
\hline
\end{tabular}


Table 3 Concentration of the glucosinolates identified in the microgreens under combined red, blue and amber LED. ${ }^{a}$ Values are expressed as $\mu \mathrm{g}$ sinigrin $^{\mathrm{b}}$, glucobrassicin ${ }^{\mathrm{c}}$ or gluconasturtiin ${ }^{\mathrm{d}}$ equivalents per g of microgreens (dry weight) (Continued)

\begin{tabular}{|c|c|c|c|c|c|c|c|c|c|}
\hline \multirow[b]{2}{*}{ Glucosinolate } & \multirow[b]{2}{*}{ LED $^{e}$} & \multicolumn{3}{|c|}{ B. juncea } & \multicolumn{3}{|l|}{ B. rapa } & \multicolumn{2}{|c|}{ R. sativus } \\
\hline & & $\mathrm{MB}$ & MG & MSF & MO & MR & PC & $\mathrm{RO}$ & $\mathbf{R R}$ \\
\hline & T5 & ND & ND & ND & $70.1^{*}$ & 66.4 & $13.1^{*}$ & ND & ND \\
\hline & T6 & ND & ND & ND & $64.0^{*}$ & $63.4^{*}$ & $40.9^{*}$ & ND & ND \\
\hline & T7 & ND & ND & ND & $63.3^{*}$ & $59.0^{*}$ & $36.4^{*}$ & ND & ND \\
\hline & T8 & ND & ND & ND & $59.3^{*}$ & $30.6^{*}$ & $14.9^{*}$ & ND & ND \\
\hline \multirow[t]{8}{*}{ Neoglucobrassicin $^{d}$} & $\mathrm{~T} 1$ & 164.9 & 228.2 & 168.0 & 1016.2 & 698.5 & 927.4 & 9.4 & ND \\
\hline & $\mathrm{T} 2$ & $180.2^{*}$ & $285.1^{*}$ & $161.7^{*}$ & $1234.1^{*}$ & $809.8^{*}$ & $1018.4^{*}$ & ND & ND \\
\hline & T3 & $205.5^{*}$ & $227.0^{*}$ & $132.2^{*}$ & $897.4^{*}$ & $656.5^{*}$ & 756.5 & ND & 4.2 \\
\hline & T4 & $134.2^{*}$ & 249.9 & $140.5^{*}$ & $1117.3^{*}$ & $644.1^{*}$ & $843.8^{*}$ & ND & ND \\
\hline & T5 & $339.3^{*}$ & $176.1^{*}$ & $126.0^{*}$ & 1468.9 & $674.0^{*}$ & $549.5^{*}$ & $3.0^{*}$ & ND \\
\hline & T6 & $128.1^{*}$ & $227.3^{*}$ & $148.4^{*}$ & $1067.6^{*}$ & $687.3^{*}$ & $1095.2^{*}$ & ND & ND \\
\hline & T7 & $212.6^{*}$ & $238.9^{*}$ & $162.1^{*}$ & 1301.3* & $670.8^{*}$ & $1135.9^{*}$ & 8.9 & 11.8 \\
\hline & T8 & $184.9^{*}$ & $150.4^{*}$ & $95.0^{*}$ & $1105.1^{*}$ & $396.8^{*}$ & $599.5^{*}$ & ND & ND \\
\hline \multirow[t]{8}{*}{ Gluconasturtiin $^{e}$} & $\mathrm{~T} 1$ & 137.8 & 115.2 & 192.7 & 339.4 & 509.5 & 355.8 & 1.8 & ND \\
\hline & $\mathrm{T} 2$ & $141.6^{*}$ & $95.6^{*}$ & $145.2^{*}$ & $219.8^{*}$ & $276.5^{*}$ & $366.3^{*}$ & $0.9^{*}$ & 1.8 \\
\hline & T3 & $190.1^{*}$ & $103.5^{*}$ & $161.0^{*}$ & $259.5^{*}$ & $433.5^{*}$ & $251.7^{*}$ & $1.6^{*}$ & 2.4 \\
\hline & T4 & $88.5^{*}$ & $142.7^{*}$ & 187.6 & $328.0^{*}$ & $498.0^{*}$ & $284.9^{*}$ & ND & 1.6 \\
\hline & T5 & $170.6^{*}$ & $69.2^{*}$ & 183.8 & 294.8 & $410.9^{*}$ & $204.6^{*}$ & $1.7^{*}$ & 1.1 \\
\hline & T6 & $93.5^{*}$ & 100.0 & $211.6^{*}$ & $284.7^{*}$ & $553.8^{*}$ & $441.8^{*}$ & $1.2^{*}$ & 1.3 \\
\hline & T7 & $85.8^{*}$ & 94.6 & $169.8^{*}$ & $190.0^{*}$ & $356.1^{*}$ & $305.8^{*}$ & $1.7^{*}$ & 3.2 \\
\hline & T8 & $76.6^{*}$ & $70.4^{*}$ & $121.1^{*}$ & $195.7^{*}$ & $152.2^{*}$ & $170.1^{*}$ & ND & 1.1 \\
\hline
\end{tabular}

MB: mustard (Barbarossa); MG: mustard (Garnet Giant); MSF: mustard (Scarlet Frills); MO: mizuna (Organic); MR: mizuna (Red Kingdom); PC: Pac choi (Red Pac); RR/RO: Radish (Red Rambo)/Organic

ND: not detected; trace: found in trace quantities; *significantly different from control $(p<0.05)$

${ }^{a-d}$ Only one extraction replica was used for quantification, as the semi-targeted analysis revealed high fidelity (RSD $<5 \%$ ) of extracted samples in all microgreens tested

e LED treatment: T1, 4.73A:20.52B:74.36R (control); T2, 6.74A:22.68B:70.25R; T3, 9.51A:24.13B:65.91R; T4, 12.02A:26.26B:61.14R; T5,12.57A:44.31B:42.39R; T6,

14.53A:30.18B:51.82R; T7, 18.45A:33.51B:47.48R; T8, 39.46A:58.94B:0.57R

glucosinolates was correlated to $A O P 2$ and $A O P 3$ gene expressions in Arabidopsis (Liu et al. 2017), suggesting these genes might be useful markers for studying the effect of LED on the biosynthesis of the health beneficial glucoraphanin in B. rapa and $R$. sativus varieties.

The accumulation of progoitrin 2 under rbaLED in the microgreens was largely species-dependent and varied. Progoitrin was found in all, but mainly in B. rapa microgreens. In fact, it was the most prevalent glucosinolate of B. rapa Microgreens (Table 1). Progoitrin was significantly increased under all rbaLED treatments in MG by $5.3-175.4 \%$ and decreased under all treatments in MSF by $3.5-56 \%$ (Table 3). Progoitrin in B. oleracea was not significantly affected by combined red, blue and green LED, but except by combined red and blue LED (Kopsell et al. 2014). Progoitrin contributes to the bitterness of the vegetables, and high intake of it may cause an enlargement of the thyroid and can interfere with thyroid function, therefore breeding effort has been made to lower this GLS in Brassica vegetables (Groenbaek et al. 2019). Our findings suggest that rbaLED can be a viable option to reduce GLS such as progoitrin in select families of microgreens (van Doorn et al. 1998).

Gluconapin 7 was overall significantly decreased under rbaLED in MB (5-28.6\%), MR (1.5-39\%), MO (3.716.9\%), MSF (23.5-50.9\%) and RO (91-96.7\%) microgreens. Conversely, gluconapin was overall significantly increased in MG (11.2-11.6\%), PC (5.2-29.2\%) and RR (83.3-5883.3\%) (Table 3). Gluconapin was significantly decreased in B. oleracea (kale) under blue $(470 \mathrm{~nm})$ LED compared to white $(440-660 \mathrm{~nm})$ LED (Kyriacou et al. 2016). Increasing the fraction of blue LED from control also significantly decreased gluconapin in most microgreens of the present study, a potential desirable outcome for consumer acceptability due to the bitter traits of this compound. White LED followed by blue and red LED significantly increased the gluconapin in B. juncea; however, prolonged photoperiod of white LED decreased 
it (Park et al. 2020). Special attention must be given to the quantity of blue-light illumination on Brassica vegetables and for the overall balanced visible light spectrum for productivity, because contrary to popular belief, white LED does not emit all wavelengths in the visible spectrum and is mostly generated by use of phosphor coating material that absorbs blue photons and luminesces at longer wavelengths (Kusuma et al. 2020).

$B$. juncea microgreens contained the least amount of glucoerucin 9 on average $(3.4 \mu \mathrm{g} / \mathrm{g})$ compared to B. rapa $(809.9 \mu \mathrm{g} / \mathrm{g})$ and $R$. sativus $(86.3 \mu \mathrm{g} / \mathrm{g})$ (Table 1). It was overall decreased under rbaLED in MO (27.6-71.9\%) and MR (19.8-48.9\%) microgreens and was variably accumulated in RO, RR PC, MB and MSF microgreens (Table 3). Under $5 \%$ blue $/ 85 \%$ red $/ 10 \%$ green LED, glucoerucin was highest compared to $20 \%$ blue/ $80 \%$ red LED treatments in broccoli microgreens, signifying the importance of supplementary green light within the $500-600 \mathrm{~nm}$ range of least photosynthetic active radiation (D. A. Kopsell et al. 2014). Results of the present study were obtained with amber $(590 \mathrm{~nm})$ light in various fractions supplementary to red and blue components, indicating that this portion of the spectrum did not generally benefit the accumulation of glucoerucin in the majority of microgreens, but rather decreased the amount. This highlights the importance of studying green light as its effects under a broad range (500-600 $\mathrm{nm}$ ) have been reported with many contrasting results on plant growth (Brazaityte et al. 2016; Kamal et al. 2020; Samuolienè et al. 2013).

Gluconapoleiferin 5 was the only GLS that was detected only in B. rapa microgreens grown under rbaLED (Table 1). Gluconapoleiferin was significantly decreased in MR (5.1-50.8\%) and PC (6.4-66.3\%), and only increased in MO (1-24.9\%) (Table 3). Compared to other classes of GLS, aliphatic gluconapoleiferin in rutabaga (B. napus) was not significantly changed by spectral qualities (white fluorescent, far-red, red and blue) or photoperiod suggesting the response of this GLS to LED was species-specific (Mølmann et al. 2020).

The accumulation of glucoalyssin 6 under rbaLED was specific to $B$. rapa and $R$. sativus. Glucoalyassin was significantly decreased in B. rapa (MO, 2.3-13.4\%; MR, 9.5-41.8\%; PC, 19.6-32.1\%) and increased in R. sativus (RO, 29.3-53.4\%; RR, 8.3-60\%) (Table 3). Interestingly, glucoalyssin was not detected in control (T1) microgreens of B. juncea (MB and $\mathrm{MG}$ ) while certain treatments slightly increased its content. It is clear that higher fractions of blue LED accumulates glucoalyssin, agreeing with findings of a recent study which observed higher contents of glucoalyssin in $B$. napus sprouts grown under monochromatic white or blue LED compared to monochromatic red LED and combined red and blue LED (Park et al. 2019). Glucoalyssin content was high in the lateral bud (leaves) of B. juncea (baby mustard) and was undetected in other parts, which could partly explain the higher density of phytochemicals of leafy microgreens by weight compared to their mature counterparts (Sun et al. 2018; Zou et al. 2021). In most cases, GLS composition is typically uniform in the same species; however, variation in profiles under LED is common and evidence is seen in B. juncea sprouts whose overall GLS contents were decreased following two weeks of exposure, regardless of the spectral treatment used (Park et al. 2020).

\section{Indole and aromatic GLS}

Indole GLS were significantly decreased across most microgreens under rbaLED. Total contents of indoles $(16943-49,093 \mu \mathrm{g} / \mathrm{g})$ were higher than aliphatic (837$24,924 \mu \mathrm{g} / \mathrm{g})$ and aromatic $(10,853 \mu \mathrm{g} / \mathrm{g})$ GLS. Glucobrassicin 10 was overall significantly decreased in $\mathrm{MB}$ (4.5-31.4\%), MG (3.2-50\%), MSF (5.7-37.9\%), MO (1.7-37.4), MR (6.9-56\%), PC (1.7-29.8\%) and RR (6$27.5 \%)$, and increased in RO (12.6-82.8\%) only (Table $3)$. Independent of the species-specific responses under light, it is not uncommon to have indole, aromatic and total GLS contents increase $200-300 \%$ under combined green, red and blue LED compared to fluorescent/incandescent light, especially for Brassica vegetables (Sams et al. 2013). Glucobrassicin in B. rapa (mustard, choy sum) was not significantly changed under different spectral qualities (red, blue, red/blue and white) or light intensity; however, a significant interaction between the light and stage of growth was observed (Tan et al. 2020). Interestingly, mean concentration of glucobrassicin under rbaLED was highest in $R$. sativus $(1425.6 \mu \mathrm{g} / \mathrm{g})$ compared to B. rapa $(770.5 \mu \mathrm{g} / \mathrm{g})$ and $B$. juncea $(324.7 \mu \mathrm{g} / \mathrm{g})$ (Table 1), while its total concentration was the highest among indole GLS. Indole-3-carbinol, a breakdown product of glucobrassicin, has been shown to modulate certain cancers in situ and inflammation in animal models (Busbee et al. 2015; Katz et al. 2018). This highlights the importance of narrowband LED for improving contents of health-promoting compounds at different stages of growth and for maximizing the accumulation of indole GLS.

The accumulation of 4-methoxyglucobrassicin $\mathbf{1 2}$ was mixed under rbaLED. It was significantly increased in MG (2.4-43.4\%), MSF (8.7-25.8\%) and MO (0.8-3.5\%), decreased in MB (0.3-25.1\%), MR (14.6-32.5\%), RO (28.1-34\%) and RR (0.9-22\%) (Table 3). Other studies have shown that 4-methoxyglucobrassicin was significantly increased in B. napus grown under monochromatic red or blue LED compared to combined red, blue and white LED, with the highest increase observed under red LED (Park et al. 2019). However, increase of 4methoxyglucobrassicin in B. juncea appears to peak after 
two weeks under red LED (Park et al. 2020). B. juncea microgreens contained, on average, the lowest content $(198.8 \mu \mathrm{g} / \mathrm{g}$ d.w.) of 4-methoxyglucobrassicin compared to the rest of the microgreens ( $B$. rapa: $293.4 \mu \mathrm{g} / \mathrm{g}$ d.w.; $R$. sativus: $320.7 \mu \mathrm{g} / \mathrm{g}$ d.w.) (Table 1). From the aforementioned studies and current study results, photoperiod becomes significantly important for modulating key GLS in the microgreens (Chen et al. 2021).

4-Hydroxyglucobrassicin $\mathbf{8}$ was significantly decreased in all microgreens (MB, 7.5-50.5\%; MSF, 6.1-25.4\%; MO, 1-44.3\%; MR, 10.9-63.2\%; PC, 8.4-48.3\%; RR, 3.8$14.2 \%$ ) except in MG and RO, which was significantly increased by $2.1-17.4 \%$ and $7.9-34.6 \%$, respectively (Table 3). 4-Hydroxyglucobrassicin was also found to reach the highest level after one week of red LED exposure in B. juncea, and decreased when further exposed (Park et al. 2020). In B. napus shoots, it was not significantly affected by different LED (Park et al. 2019; Tan et al. 2020). B. rapa microgreens in the current study contained, on average, the lowest content $(181.2 \mu \mathrm{g} / \mathrm{g}$ d.w.) of 4-hydroxyglucobrassicin compared to the rest of the microgreens (B. juncea: $243.2 \mu \mathrm{g} / \mathrm{g}$ d.w.; $R$. sativus: $495.6 \mu \mathrm{g} / \mathrm{g})$ (Table 1).

For neoglucobrassicin 13, a generally negative response was observed in the rbaLED system, which resulted in the significant decrease in MG (0.4-34.1\%), MSF (3.5-43.5\%), MR (1.6-43.2\%) and RO (68.1\%), but significantly increased in $\mathrm{MB}(9.3-105.8 \%)$ and $\mathrm{MO}$ (5.1-28.1\%) and the response was variable in PC (Table 3). Neoglucobrassicin was not found in RR microgreens in the control group; however, it was accumulated under T3 and T7, albeit at low concentrations. Others have reported that neoglucobrassicin was highest in shoot tissues of B. oleracea (broccoli) under $20 \%$ blue/ $/ 80 \%$ red LED treatment, but significantly lower under fluorescent/incandescent light and 20\%blue $/ 70 \%$ red $/ 10 \%$ green LED (Kopsell et al. 2014). Neoglucobrassicin in B. napus (canola) sprouts grown under red or blue LED was not significantly different from one another; however, contents were significantly higher than sprouts grown under white LED or combined red and blue LED (Park et al. 2019). It is apparent that regulation of neoglucobrassicin synthesis by LED is species-specific; however, the microgreens in the current study do not seem to follow a similar trend as the literature and no general conclusions can be made on this GLS under the lighting. The MYB transcription factors in Brassica vegetables are maintained during biosynthesis and are divided into 2 groups, those that control high aliphatic GLS and others controlling high indole GLS (Chun et al. 2018). Thus, these transcription factors can be controlled by LED to modulate levels of certain GLS of interest. Since plants generally respond to environmental stresses such as light, the concentrations of these health-promoting components can be increased to serve as a defense response. The genes controlling the formation of the GLS would be the logical approach to challenge for the development of species-specific LED systems and for tailored-compound synthesis in future studies.

Gluconasturtiin $\mathbf{1 1}$ was the predominate aromatic GLS confirmed in the microgreens and was significantly decreased in most microgreens (MB, 32.1-44.4\%; MG, 10.2-39.9\%; MSF, 11.9-37.2\%; MO, 16.1-44\%; MR, 2.370.1\%; PC, 14.1-52.2\%; RO, 5.6-50\%), except in RR where gluconasturtiin was accumulated under all treatments but the control group (Table 3). Overall, $R$. sativus microgreens had the lowest mean concentration of gluconasturtiin $(1.6 \mu \mathrm{g} / \mathrm{g})$ under rbaLED compared to $B$. rapa $(320.1 \mu \mathrm{g} / \mathrm{g})$ and $B$. juncea $(131.2 \mu \mathrm{g} / \mathrm{g})$ (Table 1$)$. Studies have shown that gluconasturtiin content in $B$. juncea was highest under two weeks of blue LED exposure (Park et al. 2020), but in B. napus it was not significantly affected (C. H. Park et al. 2019). Broccoli microgreens exposed to $100 \%$ blue LED five-days before harvest significantly increased of gluconasturtiin which indicates the importance of spectral quality and photoperiod on this compound that is shown to be healthpromoting by inducing oxidative damage to human cancer cells (Kopsell et al. 2014; Soundararajan and Kim 2018). From the available literature data on gluconasturtiin response to LED in Brassica vegetables, photoperiod is equally important and should be closely examined to optimize conditions.

\section{Conclusions}

Results of the present study demonstrated that amber LED in combination with blue and red lights contributed to the altered GLS profile and increase and/or decrease in quantity of certain GLS, particularly the aliphatic GLS. The effect of rbaLED is species-specific among the eight Brassica microgreens tested, which suggests that the development of microgreens with high concentrations of GLS using the LED technology may be multifaceted. In addition to species-specific responses, spectral qualities played a large role in the profile and quantity of the GLS detected. Under rbaLED overall, $R$. sativus (RR and RO) and MG microgreens contained the highest profile of GLS while PC, MG and RR the lowest. Key features among the detected GLS include sinigrin, which was increased in most microgreens under rbaLED lighting; glucoalyssin, uniquely increasing in $R$. sativus and decreasing in B. rapa; and glucobrassicin uniquely decreasing in B. rapa and B. juncea. Gluconasturtiin was decreased in most microgreens under rbaLED and was the least concentrated GLS in $R$. sativus.

The utilization of LED technology in controlled environments for vegetable production in is an emerging 
field of modern farming, thus further investigation is warranted under novel and/or underutilized wavelengths and intensities to produce highly nutritious microgreen vegetables across a broad spectrum. Depending on consumer preference, the LED technology may also be used in lowering unwanted bitterness of certain microgreen vegetables. The literature is very limited on the effects of LED light on gene expression in the biosynthetic pathways and only a few to date have been studied in microgreens. Also, to understand the molecular mechanisms of light-regulated GLS biosynthesis, studies must consider the level of co-expression with sulphate assimilation since sulfur is essential for the primary and secondary metabolism of the plant as well as a donor for cysteine and methionine precursors (Huseby et al., 2013). Genes controlling enzymes in the biosynthetic pathway and their modulatory response under LED, for example, would provide a more logical understanding of individual compound synthesis.

\section{Abbreviations}

GLS: glucosinolate(s); LED: light-emitting diodes; AL: amber light; RL: red light; BL: blue light; rba: red/blue/amber; MO: mizuna (organic); MR: mustard, red kingdom; PC: Pac choi; RR/RO: radish red Rambo/organic; MSF: mustard Scarlet Frills; MB: mustard Barbarossa; MG: mustard Garnet giant; QC: quality control; MS: mass spectrometry

\section{Supplementary Information}

The online version contains supplementary material available at https://doi. org/10.1186/s43014-021-00072-y.

Additional file 1: Supplementary Table S1. LED lighting treatments used for growing eight Brassica microgreens under various ratios of amber (A), blue (B) and red (R) light.

Additional file 2: Supplementary Table S2. Limit of detection (LOD) and limit of quantitation (LOQ) of three standards of glucosinolates (sinigrin, glucobrassicin and gluconasturtiin) in each set of microgreens measured in $\mu \mathrm{g} / \mathrm{mL}$.

\section{Acknowledgements}

This study was supported by the A-base funds of Agriculture \& Agri-food Canada (AAFC). Project \#J-001328.001.04 and \#J-002228.001.07. The authors appreciate the Harrow Research \& Development Center (AAFC) for the use of the facilities to carry out the experiments.

\section{Authors' contributions}

O.A. performed the experiments, data analysis and write-up of the manuscript. L.M. for mass spectrometry operations, R.L for review of manuscript draft, X.H., M.F.M and R.T. for conceptualization, supervision, review and final edit of manuscript. All authors read and approved the final manuscript.

\section{Funding}

Not applicable

Availability of data and materials

All necessary data is included in this paper.

\section{Declarations}

Ethics approval and consent to participate Not applicable.

\section{Consent for publication}

All authors consent to the publication of the manuscript.

\section{Competing interests}

The authors declare no competing financial interest.

\section{Author details}

${ }^{1}$ Guelph Research \& Development Center, Agriculture and Agri-Food Canada, 93 Stone Road West, Guelph, Ontario N1G 5C9, Canada. ${ }^{2}$ Department of Food Science, Ontario Agricultural College, University of Guelph, Guelph, Ontario N1G 2W1, Canada. ${ }^{3}$ Harrow Research \& Development Center, Agriculture and Agri-Food Canada, 2585 County Road 20, Harrow, Ontario NOR 1G0, Canada.

Received: 30 June 2021 Accepted: 29 August 2021

Published online: 18 October 2021

\section{References}

Alrifai, O., Hao, X., Liu, R., Lu, Z., Marcone, M. F., \& Tsao, R. (2020). Amber, red and blue LEDs modulate phenolic contents and antioxidant activities in eight cruciferous microgreens. Journal Food Bioactives, 11(1), 1-15. https://doi.org/1 $0.31665 / J F B .2020 .11241$

Alrifai, O., Hao, X., Liu, R., Lu, Z., Marcone, M. F., \& Tsao, R. (2021). LED-induced carotenoid synthesis and related gene expression in Brassica microgreens. Journal of Agricultural and Food Chemistry, 69(16), 4674-4685. https://doi. org/10.1021/acs.jafc.1c00200.

Au-Grosser, K., \& Au-van-Dam, N. M. (2017). A straightforward method for Glucosinolate extraction and analysis with high-pressure liquid chromatography (HPLC). Journal of Visualized Experiments, 121, e55425. https://doi.org/10.3791/55425,121.

Barba, F. J., Nikmaram, N., Roohinejad, S., Khelfa, A., Zhu, Z., \& Koubaa, M. (2016) Bioavailability of Glucosinolates and their breakdown products: Impact of processing. Frontiers in Nutrition, 3, 24-24. https://doi.org/10.3389/fnut.2016. 00024

Bhandari, S. R., Rhee, J., Choi, C. S., Jo, J. S., Shin, Y. K., \& Lee, J. G. (2020). Profiling of individual Desulfo-Glucosinolate content in cabbage head (Brassica oleracea var. capitata) germplasm. Molecules (Basel, Switzerland), 25(8), 1860 https://doi.org/10.3390/molecules25081860.

Bialecki, J. B., Ruzicka, J., Weisbecker, C. S., Haribal, M., \& Attygalle, A. B. (2010). Collision-induced dissociation mass spectra of glucosinolate anions. Journal of Mass Spectrometry, 45(3), 272-283. https://doi.org/10.1002/jms.1711.

Brazaitytè, A., Viršilè, A., Samuoliene, G.J.J, , Sakalauskienè, S., Sirtautas, R., .. Duchovskis, P. (2016). Light quality: Growth and nutritional value of microgreens under indoor and greenhouse conditions (Vol. 1134).

Busbee, P. B., Nagarkatti, M., \& Nagarkatti, P. S. (2015). Natural indoles, Indole-3Carbinol (I3C) and 3,3'-Diindolylmethane (DIM), attenuate staphylococcal enterotoxin B-mediated liver injury by downregulating miR-31 expression and promoting Caspase-2-mediated apoptosis. PLoS One, 10(2), e0118506 https://doi.org/10.1371/journal.pone.0118506.

Cartea, M. E., \& Velasco, P. (2008). Glucosinolates in Brassica foods: Bioavailability in food and significance for human health. Phytochemistry Reviews, 7(2), 213229. https://doi.org/10.1007/s11101-007-9072-2.

Carvalho, S. D., \& Folta, K. M. (2014). Sequential light programs shape kale (Brassica napus) sprout appearance and alter metabolic and nutrient content. Horticulture Research, 1(1), 8. https://doi.org/10.1038/hortres.2014.8.

Chen, J., Chen, Z., Li, Z., Zhao, Y., Chen, X., Wang-Pruski, G., \& Guo, R. (2021). Effect of photoperiod on Chinese kale (Brassica alboglabra) sprouts under white or combined red and blue light. Frontiers in Plant Science, 11(2098). https://doi. org/10.3389/fpls.2020.589746.

Choi, J. Y., Desta, K. T., Lee, S. J., Kim, Y.-H., Shin, S. C., Kim, G.-S., ... Abd El-Aty, A. M. (2018). LC-MS/MS profiling of polyphenol-enriched leaf, stem and root extracts of Korean Humulus japonicus Siebold \& Zucc and determination of their antioxidant effects. Biomedical Chromatography, 32(5), e4171. https://doi. org/10.1002/bmc.4171.

Chun, J.-H., Kim, N.-H., Seo, M.-S., Jin, M., Park, S. U., Arasu, M. V., ... Al-Dhabi, N. A. (2018). Molecular characterization of glucosinolates and carotenoid biosynthetic genes in Chinese cabbage (Brassica rapa L. ssp. pekinensis). Saudi Journal of Biological Sciences, 25(1), 71-82. https://doi.org/10.1016/j. sjbs.2016.04.004.

Clarke, D. B. (2010). Glucosinolates, structures and analysis in food. Analytical Methods, 2(4), 310-325. https://doi.org/10.1039/B9AY00280D. 
Dekić, M. S., Radulović, N. S., Stojanović, N. M., Randjelović, P. J., Stojanović-Radić, Z. Z., Najman, S., \& Stojanović, S. (2017). Spasmolytic, antimicrobial and cytotoxic activities of 5-phenylpentyl isothiocyanate, a new glucosinolate autolysis product from horseradish (Armoracia rusticana P. Gaertn., B. Mey. \& Scherb., Brassicaceae). Food Chemistry, 232, 329-339. https://doi.org/10.1016/j. foodchem.2017.03.150.

El Sayed, A. M., Basam, S. M., El-Naggar, E.-M. B. A., Marzouk, H. S., \& El-Hawary, S. (2020). LC-MS/MS and GC-MS profiling as well as the antimicrobial effect of leaves of selected Yucca species introduced to Egypt. Scientific Reports, 10(1), 17778. https://doi.org/10.1038/s41598-020-74440-y.

Fahey, J. W., Zalcmann, A. T., \& Talalay, P. G. (2001). The chemical diversity and distribution of glucosinolates and isothiocyanates among plants, The chemical diversity and distribution of glucosinolates and isothiocyanates among plants.

Groenbaek, M., Kidmose, U., Tybirk, E., \& Kristensen, H. L. (2019). Glucosinolate content and sensory evaluation of baby leaf rapeseed from annual and biennial white- and yellow-flowering cultivars with repeated harvesting in two seasons. Journal of Food Science, 84(7), 1888-1899. https://doi.org/1 $0.1111 / 1750-3841.14680$

Hahn, C., Müller, A., Kuhnert, N., \& Albach, D. (2016). Diversity of kale (Brassica oleracea var. sabellica): Glucosinolate content and phylogenetic relationships. Journal of Agricultural and Food Chemistry, 64(16), 3215-3225. https://doi. org/10.1021/acs.jafc.6b01000.

Hooshmand, K., \& Fomsgaard, I. S. (2021). Analytical methods for quantification and identification of intact Glucosinolates in Arabidopsis roots using LC-QqQ(LIT)-MS/MS. Metabolites, 11(1), 18. https://doi.org/1 0.3390/metabo11010047.

Kamal, K. Y., Khodaeiaminjan, M., El-Tantawy, A. A., Moneim, D. A., Salam, A. A., Ash-shormillesy, S. M. A. I., ... Ramadan, M. F. (2020). Evaluation of growth and nutritional value of Brassica microgreens grown under red, blue and green LEDs combinations. Physiologia Plantarum, 169(4), 625-638. https://doi. org/10.1111/ppl.13083.

Katz, E., Nisani, S., \& Chamovitz, D. A. (2018). Indole-3-carbinol: A plant hormone combatting cancer. F1000Res, 7. https://doi.org/10.12688/f1000research.1412 7.1 .

Keskes, H., Belhadj, S., Jlail, L., El Feki, A., Damak, M., Sayadi, S., \& Allouche, N. (2017). LC-MS-MS and GC-MS analyses of biologically active extracts and fractions from Tunisian Juniperus phoenice leaves. Pharmaceutical Biology, 55(1), 88-95. https://doi.org/10.1080/13880209.2016.1230139.

Kim, H. J., Lee, M. J., Jeong, M. H., \& Kim, J. E. (2017). Identification and quantification of Glucosinolates in kimchi by liquid chromatographyelectrospray tandem mass spectrometry. International Journal of Analytical Chemistry, 2017, 6753481-6753488. https://doi.org/10.1155/2017/6753481.

Kopsell, D., \& Sams, C. (2013). Increases in shoot tissue pigments, Glucosinolates, and mineral elements in sprouting broccoli after exposure to short-duration blue light from light emitting diodes. Journal of the American Society for Horticultural Science, 138(1), 31-37. https://doi.org/10.21273/JASHS.138.1.31.

Kopsell, D. A., Sams, C. E., Barickman, T. C., \& Morrow, R. C. (2014). Sprouting broccoli accumulate higher concentrations of nutritionally important metabolites under narrow-band light-emitting diode lighting. Journal of the American Society for Horticultural Science J. Amer. Soc. Hort. Sci.. https://doi. org/10.21273/jashs.139.4.469, 139, 4, 469, 477

Kopsell, D. A., Sams, C. E., \& Morrow, R. C. (2015). Blue wavelengths from LED lighting increase nutritionally important metabolites in specialty crops. HortScience, 50(9), 1285-1288. https://doi.org/10.21273/hortsci.50.9.1285.

Kusuma, P., Pattison, P. M., \& Bugbee, B. (2020). From physics to fixtures to food: Current and potential LED efficacy. Horticulture Research, 7(1), 56. https://doi. org/10.1038/s41438-020-0283-7.

Kyriacou, M. C., Rouphael, Y., Di Gioia, F., Kyratzis, A., Serio, F., Renna, M., .. Santamaria, P. (2016). Micro-scale vegetable production and the rise of microgreens. Trends in Food Science \& Technology, 57, 103-115. https://doi. org/10.1016/j.tifs.2016.09.005.

Lee, M., Valan Arasu, M., Park, S., Byeon, D., Chung, S.-O., Park, S. U., ... Sun, J. (2016). LED lights enhance metabolites and antioxidants in Chinese cabbage and kale. Brazilian Archives of Biology and Technology, e16150546, 59(0). https://doi.org/10.1590/1678-4324-2016150546.

Lefsrud, M., Kopsell, D. a., \& Sams, C. E. (2008). Irradiance from distinct wavelength light-emitting diodes affect secondary metabolites in kale. HortScience, 43(7), 2243-2244. https://doi.org/10.21273/HORTSCI.43.7.2243.

Liu, Z., Hirani, A. H., McVetty, P. B., Daayf, F., Quiros, C. F., \& Li, G. (2012). Reducing progoitrin and enriching glucoraphanin in Brassica napus seeds through silencing of the GSL-ALK gene family. Plant Molecular Biology, 79(1-2), 179 189. https://doi.org/10.1007/s11103-012-9905-2.

Liu, Z., Liang, J., Zheng, S., Zhang, J., Wu, J., Cheng, F., ... Wang, X. (2017). Enriching glucoraphanin in Brassica rapa through replacement of BrAOP2.2/ BrAOP2.3 with non-functional genes. Frontiers in Plant Science, 8(1329). https://doi.org/10.3389/fpls.2017.01329.

Maina, S., Misinzo, G., Bakari, G., \& Kim, H.-Y. (2020). Human, animal and plant health benefits of Glucosinolates and strategies for enhanced bioactivity: A systematic review. Molecules (Basel, Switzerland), 25(16), 3682. https://doi. org/10.3390/molecules25163682.

Maldini, M., Baima, S., Morelli, G., Scaccini, C., \& Natella, F. (2012). A liquid chromatography-mass spectrometry approach to study "glucosinoloma" in broccoli sprouts. Journal of Mass Spectrometry, 47(9), 1198-1206. https://doi org/10.1002/jms.3028.

Mazumder, A., Dwivedi, A., \& du Plessis, J. (2016). Sinigrin and its therapeutic benefits. Molecules (Basel, Switzerland), 21(4), 416. https://doi.org/10.3390/ molecules21040416.

Mølmann, J. A., Hansen, E., \& Johansen, T. J. (2020). Effects of supplemental LED light quality and reduced growth temperature on swede (Brassica napus L ssp. rapifera Metzg.) root vegetable development and contents of glucosinolates and sugars. Journal of the Science of Food and Agriculture, 101(6), 2422-2427. https://doi.org/10.1002/jsfa.10866.

Moon, J., Jeong, J. M., Lee, S. I., Lee, J. G., Hwang, H., Yu, J., ... Kim, J. (2015). Effect of LED mixed light conditions on the glucosinolate pathway in brassica rapa. Journal of Plant Biotechnology, 42(3), 245-256. https://doi.org/10.5010/JPB.201 5.42.3.245.

Park, C. H., Kim, N. S., Park, J. S., Lee, S. Y., Lee, J. W., \& Park, S. U. (2019). Effects of light-emitting diodes on the accumulation of Glucosinolates and phenolic compounds in sprouting canola (Brassica napus L.). Foods, 8(2), 76. https:// doi.org/10.3390/foods8020076.

Park, C. H., Park, Y. E., Yeo, H. J., Kim, J. K., \& Park, S. U. (2020). Effects of lightemitting diodes on the accumulation of phenolic compounds and Glucosinolates in Brassica juncea sprouts. Horticulturae, 6(4), 77. https://doi. org/10.3390/horticulturae6040077.

Rangkadilok, N., Nicolas, M. E., Bennett, R. N., Premier, R. R., Eagling, D. R., \& Taylor, P. W. J. (2002). Determination of sinigrin and glucoraphanin in Brassica species using a simple extraction method combined with ion-pair HPLC analysis. Scientia Horticulturae, 96(1), 27-41. https://doi.org/10.1016/50304-423 8(02)00119-X.

Rechner, O., Neugart, S., Schreiner, M., Wu, S., \& Poehling, H.-M. (2017). Can narrow-bandwidth light from UV-A to green alter secondary plant metabolism and increase Brassica plant defenses against aphids? PLoS One, 12(11), 20. https://doi.org/10.1371/journal.pone.0188522.

Sams, C., Kopsell, D., \& Morrow, R. (2013). Glucosinolate Concentrations of Broccoli Microgreens Are Greater under Specific Narrow Wavelength LED Light Regimes than under Conventional Fluorescent/Incandescent Light in Controlled Environments. Paper presented at the 2013 ASHS Annual Conference Springs Salon A/B (Desert Springs J.W Marriott Resort).

Samuolienè, G., Brazaitytè, A., Jankauskienè, J., Viršilè, A., Sirtautas, R., Novičkovas, A., ... Duchovskis, P. (2013). LED irradiance level affects growth and nutritional quality of Brassica microgreens. Central European Journal of Biology, 8(12), 1241-1249. https://doi.org/10.2478/s11535-013-0246-1.

Shi, H., Zhao, Y., Sun, J., Yu, L., \& Chen, P. (2017). Chemical profiling of glucosinolates in cruciferous vegetables-based dietary supplements using ultra-high performance liquid chromatography coupled to tandem high resolution mass spectrometry. Journal of Food Composition and Analysis, 61, 67-72. https://doi.org/10.1016/j.jfca.2017.01.018.

Snowden, M. C., Cope, K. R., \& Bugbee, B. (2016). Sensitivity of seven diverse species to blue and green light: Interactions with photon flux. PLoS One, 11(10), e0163121. https://doi.org/10.1371/journal.pone.0163121.

Sotelo, T., Lema, M., Soengas, P., Cartea, M. E., \& Velasco, P. (2015). In vitro activity of Glucosinolates and their degradation products against Brassica-pathogenic Bacteria and Fungi. Applied and Environmental Microbiology, 81(1), 432-440. https://doi.org/10.1128/aem.03142-14.

Soundararajan, P., \& Kim, J. S. (2018). Anti-carcinogenic Glucosinolates in cruciferous vegetables and their antagonistic effects on prevention of cancers. Molecules (Basel, Switzerland), 23(11), 21. https://doi.org/10.3390/ molecules23112983.

Sun, B., Tian, Y.-X., Jiang, M., Yuan, Q., Chen, Q., Zhang, Y., ... Tang, H.-R. (2018). Variation in the main health-promoting compounds and antioxidant activity of whole and individual edible parts of baby mustard (Brassica juncea var. 
gemmifera). RSC Advances, 8(59), 33845-33854. https://doi.org/10.1039/C8RA 05504A.

Tan, W. K., Goenadie, V., Lee, H. W., Liang, X., Loh, C. S., Ong, C. N., \& Tan, H. T. W. (2020). Growth and glucosinolate profiles of a common Asian green leafy vegetable, Brassica rapa subsp. chinensis var. parachinensis (choy sum), under LED lighting. Scientia Horticulturae, 261, 108922, 261, 108922. https:/ doi.org/10.1016/j.scienta.2019.108922.

van Doorn, H. E., van der Kruk, G. C., van Holst, G.-J., Raaijmakers-Ruijs, N. C. M. E., Postma, E., Groeneweg, B., \& Jongen, W. H. F. (1998). The glucosinolates sinigrin and progoitrin are important determinants for taste preference and bitterness of Brussels sprouts. Journal of the Science of Food and Agriculture, 78(1), 30-38. https://doi.org/10.1002/(SICl) 1097-0010(199809)78:1<30:AIDJSFA79>3.0.CO;2-N.

Wang, J., Mao, S., Wu, Q., Yuan, Y., Liang, M., Wang, S., ... Wu, Q. (2021). Effects of LED illumination spectra on glucosinolate and sulforaphane accumulation in broccoli seedlings. Food Chemistry, 356, 129550. https://doi.org/10.1016/j. foodchem.2021.129550

Yan, Z., Zuo, J., Zhou, F., Shi, J., Xu, D., Hu, W., ... Wang, Q. (2020). Integrated analysis of transcriptomic and Metabolomic data reveals the mechanism by which LED light irradiation extends the postharvest quality of Pak-choi (Brassica campestris L. ssp. chinensis (L.) Makino var. communis Tsen et Lee). Biomolecules, 10(2), 252. https://doi.org/10.3390/biom10020252.

Yang, Y., Hu, Y., Yue, Y., Pu, Y., Yin, X., Duan, Y., ... Yang, Y. (2020). Expression profiles of glucosinolate biosynthetic genes in turnip (Brassica rapa var. rapa) at different developmental stages and effect of transformed flavin-containing monooxygenase genes on hairy root glucosinolate content. Journal of the Science of Food and Agriculture, 100(3), 1064-1071. https://doi.org/10.1002/ jsfa.10111.

Yu, Q., Tsao, R., Chiba, M., \& Potter, J. (2007). Oriental mustard bran reduces Pratylenchus penetrans on sweet corn. Canadian Journal of Plant Pathology, 29(4), 421-426. https://doi.org/10.1080/07060660709507488.

Zhang, Y. (2010). Allyl isothiocyanate as a cancer chemopreventive phytochemical. Molecular Nutrition \& Food Research, 54(1), 127-135. https:// doi.org/10.1002/mnfr.200900323.

Zou, L., Tan, W. K., Du, Y., Lee, H. W., Liang, X., Lei, J., ... Ong, C. N. (2021). Nutritional metabolites in Brassica rapa subsp. chinensis var. parachinensis (choy sum) at three different growth stages: Microgreen, seedling and adult plant. Food Chemistry, 357, 129535. https://doi.org/10.1016/j.foodchem.2 021.129535.

Zuluaga, D. L., Graham, N. S., Klinder, A., van Ommen Kloeke, A. E. E., Marcotrigiano, A. R., Wagstaff, C., ... Aarts, M. G. M. (2019). Overexpression of the MYB29 transcription factor affects aliphatic glucosinolate synthesis in Brassica oleracea. Plant Molecular Biology, 101(1), 65-79. https://doi.org/10.1 007/s11103-019-00890-2.

\section{Publisher's Note}

Springer Nature remains neutral with regard to jurisdictional claims in published maps and institutional affiliations.

Ready to submit your research? Choose BMC and benefit from:

- fast, convenient online submission

- thorough peer review by experienced researchers in your field

- rapid publication on acceptance

- support for research data, including large and complex data types

- gold Open Access which fosters wider collaboration and increased citations

- maximum visibility for your research: over $100 \mathrm{M}$ website views per year

At BMC, research is always in progress.

Learn more biomedcentral.com/submissions 